XVI.

\title{
Über die Paralyse des Nervus abducens bei Otitis.
}

Von

Prof. G. Gradenigo in Turin.

Im Jahre 1904 habe ich auf einen eigentümlichen Komplex von Krankheitserscheinungen hingewiesen, den ich zu jener Zeit in kurzen Intervallen, in mehreren ganz charakteristischen Fällen zu beobachten Gelegenheit hatte. Auch konnte ich einige wenige in der Litteratur vorhandene Fälle zusarnmenstellen.

Das Krankheitsbild besteht im wesentlichen in einer akuten Mittelohrentzündung mit sehr intensiven Schmerzen in der Temporal- und Scheitelgegend der gleichen Seite und im Auftreten, gewöhnlich einige Wochen nach dem Beginn der Ohrentzündung, einer Parese oder Paralyse des nervus abducens, gleichfalls derselben Seite. In der Mehrzahl der Fälle hat die Krankheit einen günstigen Ausgang ohne daß ein chirurgischer Eingriff notwendig sei. Obgleich wegen Mangels an pathologisch-anatomischem Materiale die Atiologie des in Rede stehenden Krankheitsbildes damals mit Sicherheit nicht festgestellt werden konnte, so habe ich doch der Hypothese Ausdruck gegeben, daß die Paralyse des 6. Gehirnnerven von einem an der Spitze der Pyramide gelegenen umschriebenen Herd von Pachy- und zuweilen Leptomeningitis, der durch Diffusion der Ohrentzïndung auf anatomisch präformierten Wegen entsteht, abhänge.

Nach meinen Arbeiten über diesen Gegenstand wurde von verschiedenen Autoren ziemlich zahlreiche Fälle von Paralyse des nervus abducens bei Otitis publizierte. Es wurden auch wichtige anatomische und pathologisch-anatomische Details aufgeklärt, so daß es wohl gerechtfertigt ist, das vorhandene Beobachtungsmaterial zu sammeln und zu ordnen, um die Modalitäten festzustellen, welche für die Krankheitserscheinungen charakteristiseh sind. 
Vor allem ist hervorzuheben, daB nicht ein jeder Fall von Parese oder Paralyse des nervus abducens, der im Verlaufe einer eitrigen Ohrentzündung oder einer endokraniellen Komplikation derselben auftritt, in das uns beschäftigende Krankheitsbild paßt und es mul in dieser Hinsicht deshalb eine exakte Unterscheidung gemacht werden. So z. B. ist bekannt, dab eine Paralyse des abducens in Gemeinschaft mit Paralyse von anderen motorisehen Augenmuskelnerven, sehr häufig bei otitischer eiteriger Leptomeningitis vorkommt und es muß daran gedacht werden, daß die Ursache der Entstehung der Paralyse des 6. Gehirnnerven in derartigen Fällen sich von denjenigen unterscheide, welche die Entwicklung derselben bei dem in Rede stehenden Krankheitsbilde hervorbringt. Dasselbe könnte man von der Lähmung der Augenmuskeln sagen, welche bei anderen endokraniellen Komplikationen, wie z. B. Gehirn- und Kleinhirnsabszessen usw. die bei Ohrentzündungen vorzukommen pflegen, in Erscheinung treten können.

Untersuchen wir nun auf Grund der bekannten Fälle die klinischen Charaktere.

Es können die Fälle in drei Kategorien eingeteilt werden. Vor allem sind die typischen Fälle zu erwähnen, in welchen in ganz deutlicher Weise die angedeuteten drei Symptome sich erkennen lassen, d. h. eitrige akute Mittelohrentzündung mit oder ohne Perforation des Trommelfelles und mit mehr oder weniger hochgradiger Beteiligung der Warzenzellen an der infektiösen Erkrankung der Trommelhöhle, Schmerzen, die namentlich in der Schläfen- und Scheitelgegend derselben Seite intensiv sind, schlieblich Paralyse des gleichseitigen Abducens, die später sich dem Krankheitsbilde zugesellt.

In einer anderen zweiten Gruppe von gleichfalls typischen Fällen treten nebst den charakteristischen Erscheinungen andere akzessorische Symptome auf, die, wie wir sehen werden, für sich selbst geeignet sind, Licht auf die Ätiologie des Krankheitsbildes zu werfen oder aber Komplikationen der Otitis, die jedoch höchstwahrscheinlich in keinem kausalen Konnex mit der Paralyse des Abducens stehen.

Wir werden diese Fälle bezeichnen als ty pisch e mit N ebensymptomen oder mit komplizierten Läsionen, die in keiner direkten Beziehung mit der Paralyse des Abducens stehen.

In einer dritten Kategorie erfolgt tödlicher Ausgang infolge 
von eitriger, diffuser Leptomenigitis, während in den ersten beiden vollständige Heilung eintritt.

Rücksichtlich der Symptomatologie bilden die Fälle der zweiten Kategorie einen Übergang zwischen jenen der ersten und der dritten.

Schlieblich müssen noch andere Fälle berïcksichtigt werden, in denen zwar auch Paralyse des Abducens als hervorragendes Symptom vorhanden ist, die aber trotzdem nicht zu dem in Rede stehenden Krankheitsbild gehören, weil die Entstehungsart der Paralyse verschieden ist.

Die Varietäten, welche die Symptomatologie in den letzten Fällen aufweist, hat mehrere Antoren dazu bestimmt anzunehmen, daß das angedentete Krankheitsbild nicht mit Recht als eine besondere nosologische Form beurteilt werden könne. Gegen diese Anschauung spricht aber die charakteristische Gruppierung der Symptome und der gleichförmige Verlauf in den typischen Fällen. Der beste Beweis aber, dab es sich um eine distinkte Affektion handelt, liegt darin, daß bedeutende Ohrenärzte erklärten, nie einen solchen Fall beobachtet zu haben, während andere in einem kurzen Zeitraume mehreren begegneten.

In der Tat ist es möglich, in den typischeu Fällen mit Sicherheit die Diagnose zu stellen.

In den nachstehenden Tabellen habe ich die bis jetzt bekannt gewordenen Fälle mit Angabe der wiehtigeren Charaktere zusammiengestellt und nach den oben aufgestellten drei Kategorien gruppiert. Einige derselben wurden von mir schon in früheren Arbeiten eingehend erörtert. 
I. Kategorie.

\begin{tabular}{|c|c|c|c|c|c|c|c|}
\hline $\begin{array}{l}\text { No. } \\
\text { Lite- } \\
\text { ratur }\end{array}$ & Autoren & $\begin{array}{l}\dot{j} \\
\dot{D} \\
0 \\
0 \\
\mathbb{E}\end{array}$ & 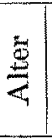 & $\frac{\$}{8}$ & $\begin{array}{l}\text { Prädispon. } \\
\text { Ursachen }\end{array}$ & $\begin{array}{l}\text { Trommelfell- } \\
\text { öffnung }\end{array}$ & $\begin{array}{l}\text { Sitz der } \\
\text { Trommel- } \\
\text { fell- } \\
\text { offfnung }\end{array}$ \\
\hline $1(5)$ & Spira 1896 & M. & 73 & L. & Influenza & $\begin{array}{l}\text { Parac. nach } \\
\text { einig. Tagen }\end{array}$ & $?$ \\
\hline $2(10)$ & Geronzi 1899 & M. & 30 & L. & Influenza & $\begin{array}{l}\text { Perfor. am } \\
\text { 2. Tag } \\
\text { Parac. am } \\
\text { 15. Tag }\end{array}$ & $?$ \\
\hline $\begin{array}{l}3(16) \\
4(17)\end{array}$ & $\begin{array}{ll}\text { Woods } 1901 \\
\text { Pisehel } 1902\end{array}$ & M. & $\begin{array}{c}? \\
94\end{array}$ & $\mathrm{~L}_{\mathrm{n}}$ & Katarrh & Parac am & $\overline{9}$ \\
\hline & & & & & $\begin{array}{l}\text { Aatamitis } \\
\text { Otitis } \\
\text { (0titis } \\
\text { bilateralis) }\end{array}$ & $\begin{array}{l}\text { farac. am } \\
\text { 9. Tag. }\end{array}$ & \\
\hline $5(36)$ & Lannois et Ferrand & M. & 38 & L. & - & Perforation & $?$ \\
\hline $6(37)$ & LubetBarbon I1904 & W. & 7 & $?$ & - & - & - \\
\hline $\begin{array}{ll}7 & (37) \\
8 & (31)\end{array}$ & Lubet BarbonII 1904 & $?$ & ? & $?$ & - & - & $\overrightarrow{9}$ \\
\hline $8(31)$ & $\begin{array}{l}\text { Gradenigo C. Lui- } \\
\text { gia } 1904\end{array}$ & W. & 31 & $\mathrm{R}$. & $\begin{array}{l}\text { Schwanger- } \\
\text { schaft im } 5 . \\
\text { Monate }\end{array}$ & $\begin{array}{l}\text { Perforation } \\
\text { am 9. Tag }\end{array}$ & ? \\
\hline $9(31)$ & id. C. Cristoforo & M. & 44 & L. & Schnupfen & $\begin{array}{l}\text { Paracentese } \\
\text { sehr spät }\end{array}$ & $?$ \\
\hline $10(31)$ & id. C. Giovanni & M. & 22 & L. & - & Nein & - \\
\hline $11(32)$ & Mongardi 1904 & M. & 62 & $\mathrm{R}$. & - & Spät & - \\
\hline $12(30 ّ)$ & Trifiletti 1904 & M. & $?$ & R. & - & Spät & $\begin{array}{l}\text { Hinten } \\
\text { unten }\end{array}$ \\
\hline $13(34)$ & Ricci 1904 & M. & 48 & L. & Pneumonie & Nein & 7 - \\
\hline $14(33)$ & Citelli I 1904 & W. & 22 & R. & - & Am 5. Tag & Zentral \\
\hline $\begin{array}{l}10 \%(43) \\
16(41)\end{array}$ & $\begin{array}{l}\text { Luc } 1905 \\
\text { Geronzi II } 1905\end{array}$ & $\begin{array}{l}\text { M. } \\
\text { M. }\end{array}$ & $\begin{array}{l}45 \\
40\end{array}$ & L. & - & 7 & Vorn \\
\hline $17(44)$ & af Forselles 1905 & M. & 11 & L. & $\begin{array}{l}\text { Halsentzuin- } \\
\text { dung }\end{array}$ & $\begin{array}{l}\text { Parac. } \\
\text { am 2. Tag }\end{array}$ & $\begin{array}{c}\text { unten } \\
?\end{array}$ \\
\hline $18(46)$ & Pick 190ä & M. & $\check{a}$ & $?$ & - & Am 3. Tag & $?$ \\
\hline $19(56)$ & Rimini III 1906 & M. & 33 & R. & - & Kleine & Vorn ant \\
\hline $20(70)$ & Ceccaroni 1907 & N. & 49 & R. & - & Am 20. Tag & Vorn un \\
\hline $21(51)$ & Terson I 1906 & W. & 12 & $\mathrm{R}$. & Ozaena & Am 20. Tag & $?$ \\
\hline $22(51)$ & Terson II 1906 & M. & $i$ & L. & Influenza & Am 4. Tag & $?$ \\
\hline $23(69)$ & Baratonx 1907 & W. & 12 & $\mathrm{~L}$ & Chronische & ? & $?$ \\
\hline $24(66)$ & Cozzolino 1907 & M. & 50 & $\mathrm{R}$. & $\begin{array}{c}\text { eitrige } \\
\text { Mittelohrent- } \\
\text { zündung. } \\
\text { Cholesteatom }\end{array}$ & - & Vom obe \\
\hline
\end{tabular}

Anmerkung. Die von Bürkner (1,18), Leimer (40) und Jack (45) 
Typische Fälle.

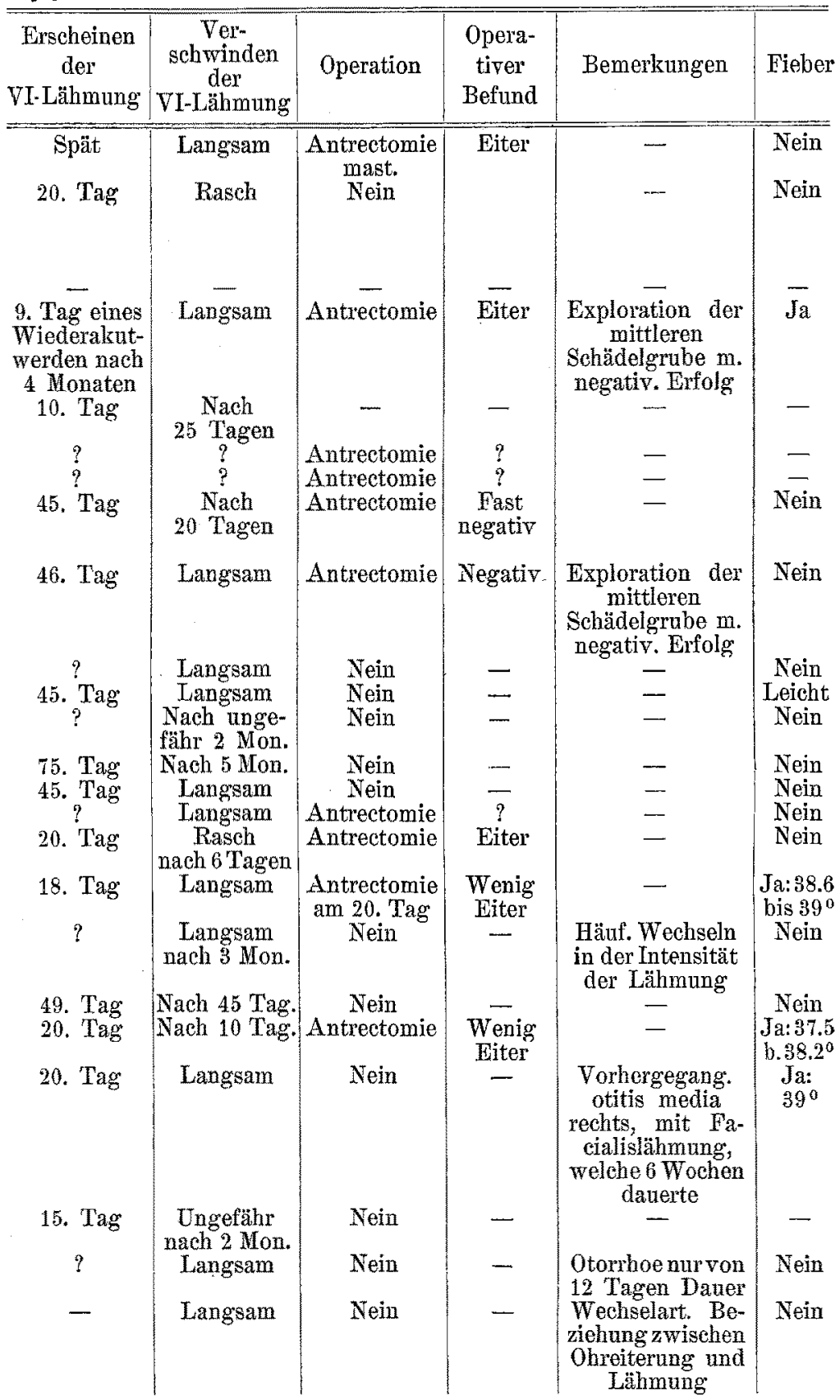

mitgeteilten Fülle sind nicht mit Sicherheit zur Syndrome zurückzuführen. 
II. Kategorie - Typische Fälle, mit a) Akute Mittel.

\begin{tabular}{|c|c|c|c|c|c|c|c|}
\hline $\begin{array}{l}\text { No. } \\
\text { Lite- } \\
\text { ratur }\end{array}$ & Autoren & 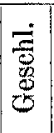 & $\stackrel{5}{\sharp}$ & 造 & $\begin{array}{c}\text { Prädispon. } \\
\text { Ursachen }\end{array}$ & $\begin{array}{l}\text { Trommelfell- } \\
\text { öffnung }\end{array}$ & $\begin{array}{c}\text { Sitz dex } \\
\text { Trommel- } \\
\text { fell } \\
\text { offundug }\end{array}$ \\
\hline $1(20)$ & Török 1903 & W. & 26 & $\mathrm{~L}$. & - & Im 8. Tag. & Vorn unt, \\
\hline $2(22)$ & Körner 1903 & W. & 12 & R. & $\begin{array}{l}\text { Wiederholte } \\
\text { Halsent- } \\
\text { zündingen }\end{array}$ & & ? \\
\hline $3(52)$ & Lombard II 1906 & M. & 8 & $\mathrm{R}$ & - & $\begin{array}{l}\text { Parac. a.2.Tg, } \\
\text { Erweit. der }\end{array}$ & ? \\
\hline $4(3)$ & Stix 1889 & M. & 21 & R. & $\begin{array}{l}\text { Recidiv. } \\
\text { Otitis }\end{array}$ & $\begin{array}{l}\text { Pertor. 1.1. } \\
\text { Spontan }\end{array}$ & $?$ \\
\hline $5(21)$ & D'Ajutolo 1903 & M. & 26 & $?$ & - & ? & ? \\
\hline $6(31)$ & $\begin{array}{l}\text { Gradenigo } 1904 \\
\text { B. Serafina }\end{array}$ & W. & 31 & $\mathrm{~L}$. & Schnupfen & $\begin{array}{l}\text { Im 3. Tag. } \\
\text { Nachh. Parac. }\end{array}$ & $\begin{array}{l}\text { Hinten } \\
\text { oben }\end{array}$ \\
\hline $7(52)$ & Lombard I 1906 & W. & 38 & $\mathrm{R}$. & Influenza & $\operatorname{Im}$ 3. Tag & Forn unt. \\
\hline $\begin{array}{l}8(24) \\
9(72)\end{array}$ & $\begin{array}{l}\text { Bonmier } 1903 \\
\text { Poppi } 1907\end{array}$ & M. & $?$ & $\begin{array}{l}\mathrm{R} . \\
\mathrm{I} \text {. }\end{array}$ & Erkälttung & $\begin{array}{l}\text { ? } \\
\text { Parac. 6. 6g. }\end{array}$ & $\stackrel{?}{\text { Vorn oben }}$ \\
\hline $10(73)$ & Modestini 1907 & M. & 30 & L. & $\begin{array}{c}\text { Erkältung } \\
\text { des Gesichts }\end{array}$ & Nach 18 Sto. & $\begin{array}{c}\text { ? } \\
?\end{array}$ \\
\hline 11. (31) & $\begin{array}{r}\text { Gradenigo } 1904 \\
\text { B. Giuseppina }\end{array}$ & W. & 17 & $\mathbf{R}$. & $\begin{array}{c}\text { Hals- } \\
\text { entzündng }\end{array}$ & $\begin{array}{l}\text { Spontan ent- } \\
\text { standene }\end{array}$ & ? \\
\hline $12(48)$ & Tommasi I 1905 & W. & 27 & L. & $\begin{array}{c}\text { Tonsillitis. } \\
\text { Wochenbett }\end{array}$ & $\begin{array}{c}\text { Spontan. } \\
\text { Nachher Er"- } \\
\text { weiterung }\end{array}$ & $\begin{array}{c}\text { Hinten } \\
\text { oben }\end{array}$ \\
\hline $13(74)$ & Tommasi II 1907 & M. & 8 & 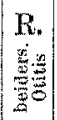 & - & $\begin{array}{l}\text { Wiederholte } \\
\text { Paracentese } \\
\text { (5 mal) }\end{array}$ & $?$ \\
\hline $14(5 \breve{a})$ & Baurowicz 1906 & W. & 16 & L. & Influenza & $\left|\begin{array}{l}\text { In den ersten } \\
24 \text { Stunden }\end{array}\right|$ & Binten \\
\hline $15(61)$ & Hastinger 1906 & M. & 22 & R. & Schnupfen & $\begin{array}{l}\text { Spontan ent- } \\
\text { stand. nach- } \\
\text { her wieder- } \\
\text { holte Parac. }\end{array}$ & $?$ \\
\hline $16(8)$ & Jugensmeyer 1898 & ? & $?$ & $?$ & - & $?$ & - \\
\hline $17(7)$ & Habermann 1898 & M. & 6 & $?$ & Bronchitis & N. wenig. Std. & $?$ \\
\hline $18(56)$ & Rimini I 1906 & W. & $i$ & R. & - & $\begin{array}{l}\text { Spontan ent- } \\
\text { standene }\end{array}$ & ? \\
\hline
\end{tabular}


Nebenerscheinungen oder mit Komplikationen.

ohrentzüudung.

\begin{tabular}{|c|c|c|c|c|c|}
\hline $\begin{array}{l}\text { Frscheinen } \\
\text { der } \\
\text { VI-Lähmung }\end{array}$ & $\begin{array}{c}\text { Ver- } \\
\text { schwinden } \\
\text { der } \\
\text { VI-Lähmung }\end{array}$ & Operation & $\begin{array}{l}\text { Opera- } \\
\text { tiver } \\
\text { Bofund }\end{array}$ & $\begin{array}{c}\text { Neben- } \\
\text { erscheinungen und } \\
\text { Komplikationen }\end{array}$ & Fiebe \\
\hline 25. Tag & Nach 35Tag. & Nein & - & & Nein \\
\hline $\begin{array}{l}\text { Ungefähr } \\
\text { 30. Tag }\end{array}$ & Langsam & $\begin{array}{c}\text { Antrectomie } \\
\text { mast. am } 12 . \\
\text { Tag }\end{array}$ & $\begin{array}{l}\text { Eiter und } \\
\text { Granul. } \\
\text { Perisin. } \\
\text { Abszeß }\end{array}$ & & Nein \\
\hline Am 19. Tag & Sehr langsam & $\begin{array}{c}\text { Antrect.Lum- } \\
\text { barpunktion } \\
\text { mit neg. Bef. }\end{array}$ & $\begin{array}{c}\text { Eiter, } \\
\text { Granula- } \\
\text { tionen }\end{array}$ & Erbrechen & $\begin{array}{l}\mathrm{Ja} \\
\text { bis } 40^{\circ}\end{array}$ \\
\hline Am 15. Tag & $\begin{array}{c}\text { Sehr langsam } \\
\text { aach unge- } \\
\text { faht } 65 \text { Tagen }\end{array}$ & Nein & & $\begin{array}{l}\text { Erbrechen. Leichte } \\
\text { Nenritis optica. } \\
\text { Nach 5 Tagen, } \\
\text { Otitis acuta links }\end{array}$ & $\mathrm{Ja}$ \\
\hline Am 30. Tag & Langsam & Antrectomie & $\underset{\text { negativ }}{\text { Fegat }}$ & $\begin{array}{l}\text { Spasmen des o } \\
\text { cularis ocul }\end{array}$ & Nein \\
\hline $\mathrm{Am} \mathrm{30.} \mathrm{Tag}$ & Langsam & $\begin{array}{l}\text { Antrectomie } \\
\text { Lumbarpunk- } \\
\text { tion zeigt ge- } \\
\text { steig. Druck }\end{array}$ & Negativ & $\begin{array}{l}\text { Orbitalschmerzen. } \\
\text { Spasmen d. orbi- } \\
\text { cularis. Erbrechen } \\
\text { Photoph. Rücken- } \\
\text { marksehmerzen }\end{array}$ & $\begin{array}{c}\mathrm{Ja} \\
\text { bis } 40\end{array}$ \\
\hline Am 40. Tag & $\begin{array}{l}\text { Mehr wie } \\
40 \text { Tage }\end{array}$ & Antrectomie & $\begin{array}{l}\text { Blasse } \\
\text { Granul. }\end{array}$ & $\begin{array}{l}\text { Pupillär } \\
\text { a. d.kra }\end{array}$ & $J_{a}$ \\
\hline 44. $\stackrel{?}{\mathrm{Tag}}$ & 78. $\mathrm{Tag}$ & $\begin{array}{l}\text { Nein } \\
\text { Nein }\end{array}$ & - & $\begin{array}{l}\text { Hyper } \\
\text { Neural }\end{array}$ & $\stackrel{?}{\text { Nein }}$ \\
\hline 40. Tag & o & Nein & & $\begin{array}{l}\text { Neuralgie } 2 \text { und } 3 \\
\text { Ast. } V\end{array}$ & Ja \\
\hline 10 & Langsam & $\begin{array}{l}\text { Antrect. Ex- } \\
\text { plor. d. mittl. } \\
\text { Schädelgrube } \\
\text { m. negat. Bef. }\end{array}$ & $\begin{array}{l}\text { Fiter und } \\
\text { Granula- } \\
\text { tionen }\end{array}$ & $\begin{array}{c}\text { VII-Lähmung. } \\
\text { Leichte Photo- } \\
\text { phobie. } \\
\text { Optische Neuritis. }\end{array}$ & Nein \\
\hline $\operatorname{Am}$ 7. Tag & Am 27. Tag & Antrectomie & $\begin{array}{l}\text { Wenig } \\
\text { Eiter, An- } \\
\text { trum }\end{array}$ & $\begin{array}{r}\text { VII-Lähn } \\
\text { 7. T }\end{array}$ & $\mathrm{Ja}$ \\
\hline Am 66. Tag & Langsam & Nein & & $\begin{array}{l}\text { VII-Parese am } \\
60 . \text { Tag }\end{array}$ & Ja \\
\hline Am 5. Tag & Am 15. Tag & Nein & - & $\begin{array}{c}\text { VII-Lähmung am } \\
\text { 2. Tag }\end{array}$ & $\mathrm{Ja}$ \\
\hline $\mathrm{Am} \mathrm{22.} \mathrm{Tag}$ & Langsam & $\begin{array}{l}\text { Antrectomie } \\
\text { am 10. Tag. } \\
\text { Radikaloper: } \\
\text { am 33. Tag }\end{array}$ & $\begin{array}{l}\text { Eiter, } \\
\text { Granula- } \\
\text { tionen }\end{array}$ & $\begin{array}{c}\text { Pyolabyrinthitis. } \\
\text { Perforat. d. ovalen } \\
\text { Fenster }\end{array}$ & $\begin{array}{c}\text { Ja } \\
\text { Leich }\end{array}$ \\
\hline ? & Nach 30 Tag. & & - & $\begin{array}{c}\text { Beiders. optische } \\
\text { Neuritis }\end{array}$ & $\mathrm{Ja}_{\mathfrak{a}}$ \\
\hline Am 40. Tag & $\begin{array}{c}\text { Mehr wie } \\
60 \text { Tage }\end{array}$ & Antrectomie & $\begin{array}{l}\text { Perisin. } \\
\text { Abszeß }\end{array}$ & $\begin{array}{l}\text { Optische Neuritis } \\
\text { Encephal. Schreien }\end{array}$ & $\mathrm{Ja}$ \\
\hline $\sin 56 . \mathrm{Tag}$ & Sehr langsam & $\begin{array}{l}\text { Antrectomie } \\
\text { im 35. Tag. } \\
\text { Spater Radi- } \\
\text { kaloperation }\end{array}$ & $\begin{array}{c}\text { Stenose d. } \\
\text { Gehör- } \\
\text { gangs d. } \\
\text { Granul. } \\
\text { Perisin. } \\
\text { AbszeB }\end{array}$ & - & Nein \\
\hline
\end{tabular}


II. Kategorie. - Typische Fälle, mit a) Akute Mittel-

\begin{tabular}{|c|c|c|c|c|c|c|c|}
\hline $\begin{array}{l}\text { No. } \\
\text { Lite- } \\
\text { ratur }\end{array}$ & Autoren & 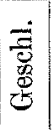 & $\stackrel{\text { 总 }}{\frac{2}{4}}$ & 总 & $\begin{array}{l}\text { Prädispon. } \\
\text { Ursachen }\end{array}$ & $\begin{array}{l}\text { Trommelfell } \\
\text { offnung }\end{array}$ & $\begin{array}{l}\text { Sitz der } \\
\text { Trommel } \\
\text { fell- } \\
\text { offunung }\end{array}$ \\
\hline $19(56)$ & Rimini II 1906 & M. & $\tau$ & L. & Scharlach & Paracentese & $?$ \\
\hline $20(54)$ & Alt 1906 & M. & 8 & L. & Influenza & Paracentese & $?$ \\
\hline $21(38)$ & Cheval 1904 & MI. & $?$ & R. & Influenza & $\begin{array}{l}\text { Spontan ent- } \\
\text { standene }\end{array}$ & $?$ \\
\hline 22 (19) & $\begin{array}{l}\text { Sturm und Suck- } \\
\text { storf } 1902\end{array}$ & W. & 12 & $R$. & - & Spontan & $?$ \\
\hline $23(6)$ & Preysing 1898 & W. & 5 & R. & - & $\begin{array}{l}\text { Paracentese } \\
\text { spät }\end{array}$ & ? \\
\hline
\end{tabular}

b) Chronische Mittel-

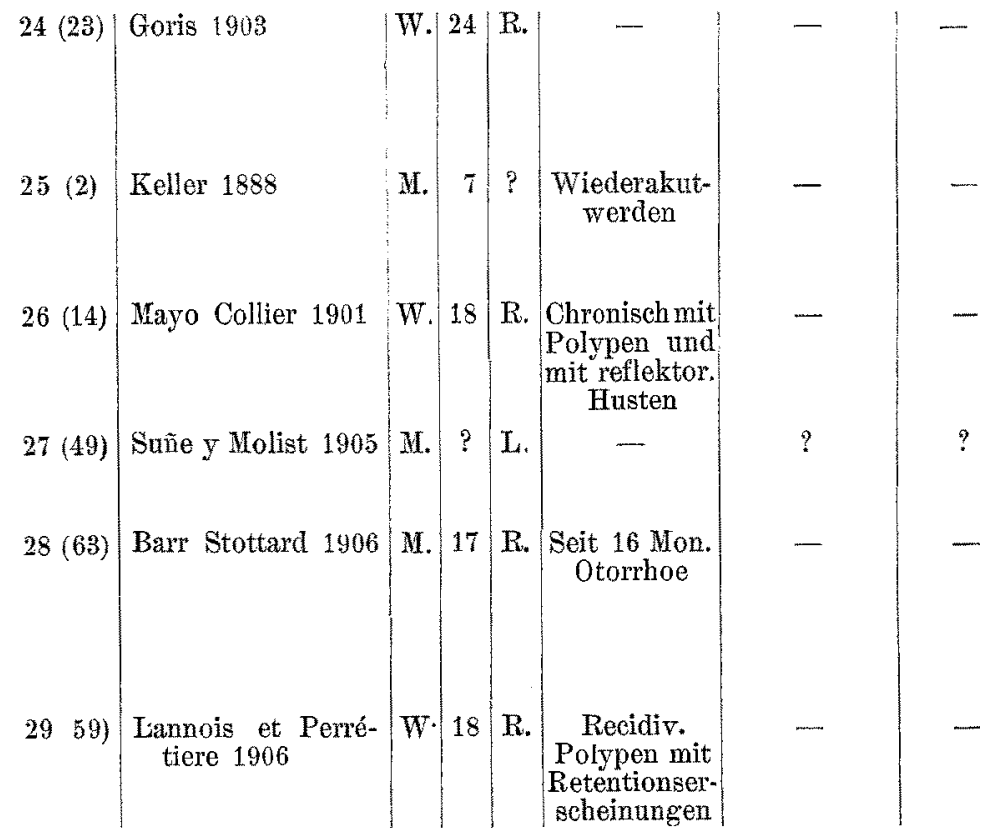


Nebenerscheinungen oder mit Komplikationen.

ohrentzündung.

\begin{tabular}{|c|c|c|c|c|c|}
\hline $\begin{array}{l}\text { Erscheinen } \\
\text { der } \\
\text { VI-Lähmung }\end{array}$ & $\begin{array}{c}\text { Ver- } \\
\text { schwinden } \\
\text { der } \\
\text { VI-Lähmung }\end{array}$ & Operation & $\begin{array}{l}\text { Opera- } \\
\text { tiver } \\
\text { Befund }\end{array}$ & $\begin{array}{c}\text { Neben- } \\
\text { erscheinungen und } \\
\text { Komplikationen }\end{array}$ & Fieber \\
\hline $\begin{array}{l}\text { ? Beiders. } \\
\text { vI Lähmung, } \\
\text { mehr links }\end{array}$ & $\begin{array}{l}\text { Schwindetzu- } \\
\text { erst d. rechts- } \\
\text { seit.Lähmung }\end{array}$ & Antrectomie & $?$ & $\begin{array}{l}\text { Arythmischer Puls, } \\
\text { Hyperämie des } \\
\text { Augen- } \\
\text { hintergrunds }\end{array}$ & $\mathrm{Ja}$ \\
\hline Am 50. Tag & Nach 7 Tagen & $\begin{array}{l}\text { Antrectomie } \\
\text { am 20. Tag }\end{array}$ & $\begin{array}{l}\text { Fistel des } \\
\text { äuB. halb- } \\
\text { zirkelfrm. } \\
\text { Kanals }\end{array}$ & Pyolabyrinthitis & $\underset{\mathrm{Ja}}{\mathrm{Ja}} 38.9^{0}$ \\
\hline Am 30. Tag & Langsam & $\begin{array}{l}\text { Nein } \\
\text { Negativ. Be- } \\
\text { fund bei Lum- } \\
\text { barpunktion }\end{array}$ & - & $\begin{array}{l}\text { Retropharyn- } \\
\text { gealer AbszeB }\end{array}$ & $\begin{array}{c}\text { Ja } \\
\text { bis } 39^{\circ}\end{array}$ \\
\hline Am 25. Tag & $\begin{array}{l}\text { Mehr wie } \\
4 \text { Monate }\end{array}$ & Antrectomie & $\begin{array}{c}\text { Eiter,Gra- } \\
\text { nul. Peris. } \\
\text { Abszeß }\end{array}$ & $\begin{array}{c}\text { Optische Neuritis } \\
\text { Erbrechen }\end{array}$ & Nein \\
\hline $\begin{array}{c}\text { Nach der } \\
\text { Antrectomie }\end{array}$ & $\begin{array}{l}\text { Nach } \\
37 \text { Tagen }\end{array}$ & Antrectomie & $\begin{array}{l}\text { Perisin. } \\
\text { Abszel }\end{array}$ & $\begin{array}{l}\text { Optische Neuritis. } \\
\text { Leichte Protrusion } \\
\text { des Auges }\end{array}$ & Ja \\
\hline
\end{tabular}

ohrentzündungen.

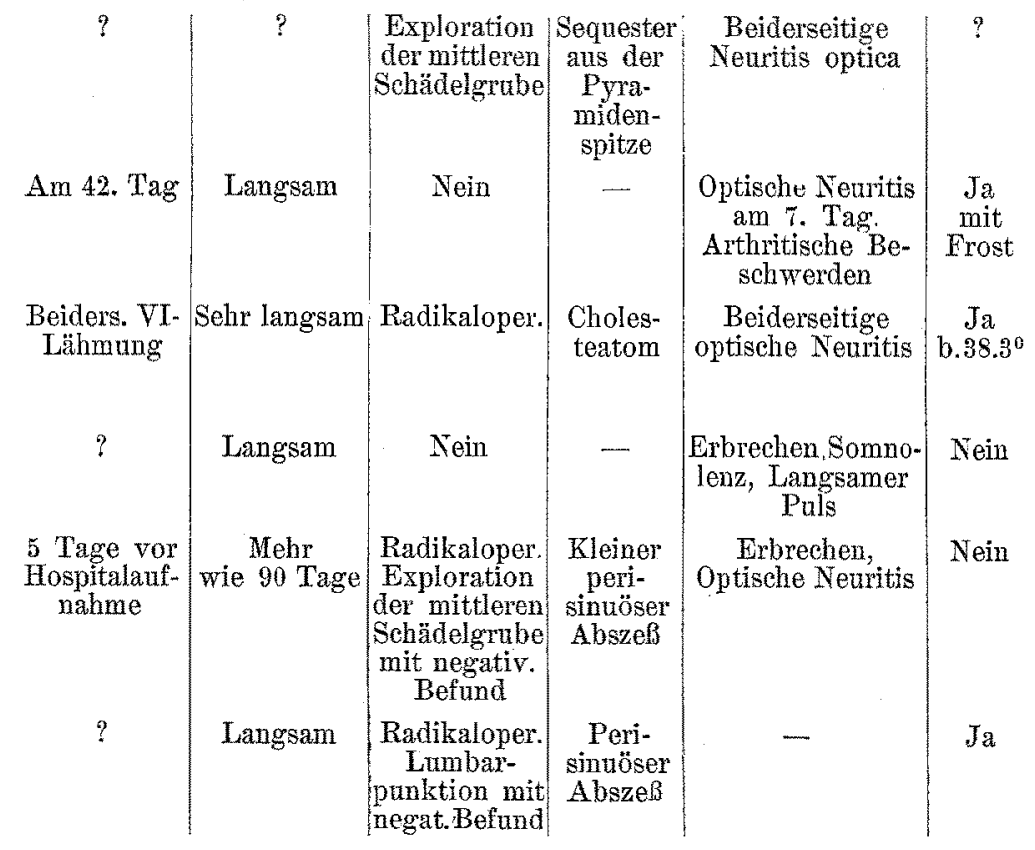


III. Kategorie. - Fälle

\begin{tabular}{|c|c|c|c|c|c|c|c|}
\hline $\begin{array}{l}\text { No. } \\
\text { Lite- } \\
\text { ratur }\end{array}$ & Autoren & $\frac{0}{5}$ & $\stackrel{5}{\frac{E}{4}}$ & 荵 & $\begin{array}{l}\text { Prädispon. } \\
\text { Ursachen }\end{array}$ & $\begin{array}{l}\text { Trommelfell- } \\
\text { öffnung }\end{array}$ & $\begin{array}{l}\text { Sitz der } \\
\text { Trommel- } \\
\text { fell- } \\
\text { offnung }\end{array}$ \\
\hline $1(31)$ & $\begin{array}{l}\text { Gradenigo } 1904 \\
\text { P. Pietro }\end{array}$ & M. & 31 & L. & Ozaena & $\begin{array}{l}\text { Sehr späte } \\
\text { Paracentese }\end{array}$ & - \\
\hline $2(50)$ & Noltenius 1905 & M. & 28 & $R$. & $\begin{array}{l}\text { Halsentzin- } \\
\text { dung }\end{array}$ & $\begin{array}{l}\text { Am 3. Tage, } \\
\text { Perforation }\end{array}$ & Vorne \\
\hline $3(58)$ & Jacques 1906 & M. & 7 & R. & $\begin{array}{c}\text { Recidivirend } \\
\text { Otitis }\end{array}$ & $\begin{array}{l}\text { Nach } 24 \text { Std. } \\
\text { Erweiterung } \\
\text { nach } 3 \text { Woch. }\end{array}$ & $?$ \\
\hline $4(67)$ & Bellotti 1907 & M. & 25 & R. & 一 & $\begin{array}{l}\text { Am 10. Tag. } \\
\text { Nachher Pa- } \\
\text { racentese }\end{array}$ & $\begin{array}{l}\text { Hinten } \\
\text { unten }\end{array}$ \\
\hline
\end{tabular}

Es handelt sich also um 57 Fälle, von denen 24 der ersten, 29 der zweiten und bloß 4 der dritten Kategorie angehören. In 7 Fällen war chronische eiterige Otitis vorhanden; es sind also überwiegend $(87 \%)$ die akuten Ohrentzündungen. Auch die Mortalität ist sehr niedrig, denn der Tod kam kaum viermal in 57 Fällen yor; in 92 Proz. ist also Heilung erfolgt, sowohl nach operativen Eingriffen in der Gegend des Warzenfortsatzes oder in der Schädelhöhle als auch ohne solche. Es kann deshalb behauptet werden, daß die Krankheit gewöhnlich zur akuten Otitis in Beziehung: steht, und daß sie in der großen Mehrzahl der Falle als gutartig angesehen werden muß. In 55 Fällen, bei welchen das Geschlecht angegeben ist, finden sich 37 Männer (67 Proz.) und nur 18 Weiber. 
mit letalem Ausgange.

\begin{tabular}{|c|c|c|c|c|c|}
\hline $\begin{array}{c}\text { Erscheinen } \\
\text { der } \\
\text { VI-Lähmung }\end{array}$ & $\begin{array}{c}\text { Ver- } \\
\text { sehwinden } \\
\text { der } \\
\text { VI-Lähmung }\end{array}$ & Operation & $\begin{array}{l}\text { Opera- } \\
\text { tiver } \\
\text { Befund }\end{array}$ & $\begin{array}{c}\text { Neben- } \\
\text { erscheinungen und } \\
\text { Komplikationen }\end{array}$ & Fieber \\
\hline $\begin{array}{l}\text { Nach } \\
\text { ungefähr } \\
90 \text { Tagen }\end{array}$ & - & $\begin{array}{l}\text { Antrectomie } \\
\text { nach } \\
21 / 2 \text { Monaten }\end{array}$ & $\begin{array}{l}\text { Fast ne- } \\
\text { gativ. Bef. } \\
\text { Explorat. } \\
\text { der mittl. } \\
\text { Schädel- } \\
\text { grube ne- } \\
\text { gativ }\end{array}$ & $\begin{array}{l}\text { Photophobie. Im } \\
\text { Lumbarpunktat } \\
\text { Streptokokken. } \\
\text { Leptomeningitis } \\
\text { diffusa } 8 \mathrm{Tg} \text {, nach } \\
\text { der Erscheinung } \\
\text { der VI-Lähmung. } \\
\text { Keine Autopsie. }\end{array}$ & Nein \\
\hline $\begin{array}{l}\text { Nach } \\
\text { 4\%/2 Monaten } \\
\text { gleichzeitig } \\
\text { mit Sym- } \\
\text { ptomen der } \\
\text { Lepto- } \\
\text { meningitis }\end{array}$ & - & $\begin{array}{l}\text { 1. Antrecto- } \\
\text { mie. } \\
\text { 2. Auslöffeln } \\
\text { von Granu- } \\
\text { lationen aus } \\
\text { der vorderen } \\
\text { Hälfte dex } \\
\text { Trommel- } \\
\text { böhle. } \\
\text { 3. Radikalop. } \\
\text { Erôffnung d. } \\
\text { Labyr. }\end{array}$ & Eiter & $\begin{array}{l}\text { Osteitis d. Pyra- } \\
\text { midenspitze, und } \\
\text { eitrige Lepto- } \\
\text { meningitis }\end{array}$ & $\mathrm{Ja}$ \\
\hline Am 30. Tag & - & Radikaloper. & $\begin{array}{l}\text { Fast ne- } \\
\text { gativ. Bef. } \\
\text { Explorat. } \\
\text { der mittl. } \\
\text { Sehädel- } \\
\text { grube mit } \\
\text { negativ. } \\
\text { Erfolg }\end{array}$ & $\begin{array}{l}\text { AusfluB v. Liquor } \\
\text { cerebrospinalis. } \\
\text { Nach } 3 \text { Wochen } \\
\text { leichte Ptosis. Lep- } \\
\text { tomening. diffusa } \\
\text { nach ungef. } 48 \text { Tg. } \\
\text { Keine Autopsie }\end{array}$ & $\mathrm{Ja}$ \\
\hline $\begin{array}{l}\text { Am 43. Tage, } \\
3 \text { Tage nach } \\
\text { Antrectomie }\end{array}$ & Langsam & Antrectomie & Viel Eiter & $\begin{array}{l}\text { Nackensteifigkeit } \\
\text { am } 15 . \text { Tag. } \\
\text { Leptomeningitis } \\
18 \text { Tage nach Ope- } \\
\text { ration. } \\
\text { Keine Autopsie }\end{array}$ & Nein \\
\hline
\end{tabular}

In 49 Fällen, in denen die Körperseite, auf welcher die Affektion auftrat, berücksichtigt wurde, kam sie 28 mal rechts, 21 links zu Stande. Es prävaliert also in leichtem Grade (57 Proz.) die Erkrankung auf der rechten Seite.

Bezüglich des Alters ergeben sich 'folgende Resultate aus 50 Fällen:

Alter Zahl der Fälle

\begin{tabular}{|c|c|c|c|c|c|}
\hline Vom & 1. & bis & zum & 10. Jahre & 12 \\
\hline ( & 2. & , & n & 20 & 10 \\
\hline$n$ & 21. & $n$ & $"$ & 30. & 12 \\
\hline$"$ & 31. & $n$ & $n$ & 40. & 9 \\
\hline
\end{tabular}




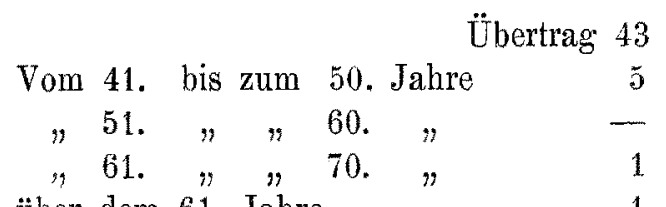

über dem 61. Jahre

\section{Gesamtzahl $=50$}

Die Erkrankung kommt also überwiegend in dem ersten Dezennien, namentlich im ersten und im dritten vor, was mit den statistischen Daten der Ohrerkrankungen überhaupt übereinstimmt.

Untersuchen wir nun genau die einzelnen Symptome.

Eiterige akute Otitis. Häufig kommen Erscheinungen einer Retention des Eiters in der Trommelhöhle wegen gänzlichen Mangels oder Insuffizienz der Perforation des Trommelfells vor. In 32 Fällen, in denen diesbezügliche Angaben vorhanden sind, ist $18 \mathrm{mal}$, also in der Hälfte der Fälle, entweder keine Perforation spontan erfolgt oder sie ist sehr spät aufgetreten, oder es wurde wohl die Parazentese vorgenommen, aber gleichfalls ziemlich spät. Die Perforation war meistens zu klein und ungenügend zu einer guten Drainage, sodaß oft die künstliche Erweiterung derselben gemacht werden mußte; anßerdem zeigte die Trommelfellöffnung oft die Neigung zum Versehlusse, sodaß die Parazentese erneut werden mußte, in einem Falle sogar 5 mal im Laufe der Krankheit (Tommasi, II Tab.).

Einen nennenswerten Umstand, auf den ich hier die Aufmerksamkeit hinlenken möchte, damit andere in Zukunft demselben Rechnung tragen möchten, bildet der relativ häufige Sitz der Perforationsöffnung in der vorderen Hälfte des Trommelfelles; denn bekanntlich kommt dies bei akuten Entzündungen nur selten vor. Unter 14 Fällen nur, in denen der Sitz der Perforation von den Autoren mit Exaktheit angegeben wird, war derselbe 8 mal, also in mehr als der Hälfte der Fälle im vorderen Teile der Membran, 5 mal im vorderen unteren, 1 mal im vorderen-oberen Segmente, 2 mal in der rorderen Hälfte. Der Parazentese oder der Erweiterung der Perforationsöffnung folgt gewöhnlich unmittelbar ein Nachlab der anderen wesentlichen Symptome, d. h. der Schmerzen und der Paralyse. Ein Fall von Pick und ein anderer yon Cozzolino sind in dieser Hinsicht höchst demonstrativ. In den chronischen Formen, welehe nur selten vorkommen und in denen es sich eigentlich um ein Akutwerden ehronischer Zustände handelt, wird die Retention des Exsudats durch Polypen oder 
durch erhärtete Eitermassen und Cholesteatomen, welche den Gehörgang verstopfen, bedingt. Im Falle von Cozzolino, auf den wir später noch zurückkommen wurden, hörte nach Entfernung jener Massen allmählich die Paralyse auf, während sie wiederum zum Vorschein kam, sobald neuerdings eine Anhäufung derselben erfolgte.

Intensive Schmerzen, namentlich in der Schläfenund Seheitelgegend derselben Seite.

Die Otitis ist von Anfang an von äußerst heftigen Schmerzen begleitet, welche kontinuierlich sind oder aber nach dem Auftreten der Eiterung aus dem Gehörgang, auf einige Tage schwinden. Die Schmerzen werden von den Kranken in das Innere des erkrankten Ohres, namentlich aber in die Schläfen- und Scheitelgegend, zuweilen auch in die Tiefe der Orbita verlegt. Auch die Bewegungen des Auges auf der Seite des erkrankten Ohres können schmerzhaft sein. Die Schmerzen müssen von denjenigen unterschieden werden, welche bei der Neuralgie des $V$. Nerven vorkommen, die als alkzessorische Erscheinung in gewissen Fällen auch in unserer Krankheit zu finden ist. Bei der Neuralgie nämlich strahlen die Schmerzen längs der Zweige des 5. Paares aus und kommen anfallsweise vor, während sie bei der in Rede stehenden Affektion intensiv und kontinuierlich sind; ron den gewöhnlichen schmerzstillenden Mitteln lassen sie sich nur wenig beeinflussen und auch nicht, wie die Schmerzen bei der Syphilis, durch antiluetische spezifische Behandlung. Zum Unterschiede von dieser letzteren zeigen sie in der Nacht keine größere Intensität.

Zuweilen mindern sich die Schmerzen und hören fast vollständig auf, wie bei der gewöhnlichen Otitis, in den ersten Tagen gleich nach dem Auftreten eines eiterigen Ausflusses und treten wieder erst nach zwei oder drei Wochen auf, als Vorläufer der Komplikation von Seiten des Auges.

Paralyse des N. abducens. Dieses Symptom scheint plötzlich, unvorhergesehen, aufzutreten: der Kranke selbst bemerkt beim Erwachen aus dem Schlafe das Doppelsehen und macht den Arzt darauf aufmerksam, der als Ursache der Diplopie sofort eine Paralyse oder Parese des N. abduzens, an der dem erkrankten Ohre entsprechenden Seite erkennt. Der Arzt könnte aber wahrscheinlich, wenn eben das Auftreten der Paralyse vorhergesehen werden könnte, durch eine systematische Kontrolle der Funktion der Augenmuskel, das gradweise Entstehen der Para- 
lyse erkennen. Es sind Fälle bekannt, in welchen der langsame Übergang einer einfachen Parese in Paralyse verfolgt werden konnte. Gewöhnlich erfolgt Heilung der Paralyse und auch diese erfolgt gradweise, zuweilen sehr langsam. Die Messungen, welche diesbezüglich von Lombard in einem seiner Fälle (II Beobachtung) gemacht worden sind, sind sehr instruktiv.

Die Paralyse tritt nur selten kurze Zeit nach dem Beginne der Otitis auf. Gewöhnlich ist sie ein spät in die Erseheinung tretendes Symptom. In 37 Fällen, in welchen mit Exaktheit die Zeit ihres Auftretens bestimmt werden konnte., erfolgte dies nur 3 mal rasch, d. h. 5-10 Tage nach dem Beginn der Otitis, 8 mal nach $15-20,20$ mal nach $20-50$, davon 10 mal nach $42-50$ Tagen. Nur ausnahmsweise tritt die Paralyse erst nach dem 56. Tage auf (6 Fälle). Man kann folglich behaupten, dab sie in der Regel ungefahr 1 oder $1 \frac{1 / 2}{2}$ Monate nach dem Beginn der akuten Ohrentzïndung auftritt.

Es wurde schon gesagt, dab die Paralyse in der großen Mehrzahl der Fälle in Heilung übergeht; das Schwinden derselben geht gewöhnlich schrittweise und allmählich, zuweilen sehr langsam vor sich. Es sind Fälle bekannt, in welchen Spuren der Paralyse noch 5 Monate nach dem Auftreten der Krankheit zu konstatieren waren (Ricci). Dies sind aber nur Ausnahmsfälle; in der Regel schwankt die Zeit, innerhalb welcher die Heilung: erfolgt, zwischen 1-3 Monaten. Wie es scheint, hört die Paralyse in jenen seltenen Fällen, in welchen sie frülizeitig zu Tage tritt, relativ rasch aut (Baurovicz: Beginn am 5., Schwund am 10. Tage; - Tommasi : Auftreten am 7., Aufhören am 20. Tage.) Regel ist dies jedoch nicht, denn es sind auch Fälle bekannt, in welchen bei raschen Auftreten der Paralyse erst nach langer Zeit das Aufhören derselben erfolgte.

Nur ausnahmsweise wurde beobachtet, dab der Paralyse Nystagmus vorausging oder daB sie von demselben begleitet worden wäre. Zwischen Parese und Paralyse kommen alle möglichen Grade vor.

Die angeführten Charaktere kennzeichnen die typischen Fälle. Von Seiten des Auges können keine anderen Symptome nachgewiesen werden: die Pupillarreaktion, der Augenhintergrund, die Funktionsfähigkeit aller Augenmuskeln, mit Ausnahme des musc. rectus ext, sind normal. Auch das Labyrinth bleibt unverletzt, sowohl im akustischen Gebiet, wie auch bezüglich des Gleichgewichts. Die Temperatur verhält sich in den typischen nicht 
komplizierten Fallen normal während der ganzen Dauer der Krankheit.

In einer zweiten Kategorie von Fällen kommen nebst den angefübrten charakteristischen Symptomen andere akzessorische vor, oder es bilden sich Komplikationen heraus. Diese letzteren scheinen nicht in ursächlichem Verhältnisse mit der Paralyse des $\mathbb{N}$. abducens zu stehen, weil sie, wie wir sehen werden, in der Yehrzahl der Fälle nicht vorhanden sind.

Die Nebenerscheinungen wie die Komplikationen vervollständigen das klinische Bild der Krankheit. 1)

Akzessorische Sym p tome. Die Erscheinungen welche nebst den erörterten drel charakteristischen Symptomen am häufigsten in den typischen Fällen erwähnt werden, deuten auf einen Reizzustand von Seiten der Gehirnbäute hin oder auf das Bestehen eines Krankheitsheerdes in der Gegend der Spitze der Pyramide.

Vor allem wird eine Entzïndung des nervus opticus getroffen: zuweilen handelt es sich hierbei um eine einfache Hyperämie der beiden Papillen, häufiger jedoch um eine wabre und zwar doppelseitige, ausnahmsweise nur einseitige Neuritis, entsprechend der erkrankten Ohrseite.

Neuritis wurde unter 33 untersuchten Fällen (1) 9 mal (ungefähr 17 Proz.) beobachtet. Dann folgen der Frequenz nach die Beobachtungen, in denen wiederholte Brechanfälle verzeichnet werden, $(8$ Fälle, also 15 Proz.). Ausnahmsweise wird Sopor, Verlangsamung des Pulses, Aufschreien in der Nacht, wie bei Leptomeningitis angegeben. Für jedes dieser letzteren Symptome wird ein Fall erwähnt. In vier Fällen, in welchen zu diagnostischen Zwecken die Lumbalpunktion ausgeführt wurde, hatte sie $3 \mathrm{mal}$ negativen Erfolg; nur einmal (Graderigo, B. Serafina) lieb sie eine erhebliche. Zunahme des Druckes des liquor cerebro-spinalis erkennen. In allen Fällen erwies sich derselbe steril,

Von den Erscheinungen von Seite des erwähnten Krankheitsherdes in der Gegend der Spitze der Felsenbeinpyramide, werden namentlich solche erwähnt, welche auf den 5 . Gehirnnerven zu beziehen sind, und zwar Hyperaesthesie, oder wahre Neuralgie (3 Fälle).

Im Falle von Bonnier war nur Hyperaesthesie im großen Gebiete des quintus vorhanden, in dem von Modestini wird

1) Es werden bei folgender Statistik die 4 Fälle mit tödlichem Ausgange infolge von Leptomeningitis nicht mitgerechnet. 
der Neuralgie des 2, und 3., in der Beobachtung von Poppi nur des 3. Astes Erwähnung getan. Außerdem werden Erscheinungen von Seiten des Okulomotorius angeführt (Verkleinerung der Pupille auf der Seite des erkrankten Ohres).

Während die Bedeutung der bis jetzt erörterten Symptome keinem Zweifel unterliegt, ist die Interpretation anderer Erscheinungen welche hic und da angeführt werden schwieriger. So z. B. wird oft das Vorhandensein von Schwindelanfällen angegeben. Dieses Symptom hängt aller Wahrseheinlichkeit nach nicht vom Labyrinthe ab, sondern steht im Zusammenhang mit der Paralyse des Abduzens und mit der davon abhängigen Diplopie. In der Tat behaupten mehrere Autoren; daB die Schwindelanfälle aufhörten sobald das erkrankte Auge verdeckt wurde. Es wird in mehreren klinischen Beobachtungen Photophobie angeführt: Diese Erscheinung könnte, wenigstens in der Mehrzahl der Fälle, als eine Konsequenz der Diplopie angesehen werden, welche namentlich in den ersten Tagen ihres Erscheinens die Kranken derart belästigt, dah sie den Aufenthalt in dunklen Räumen vorziehen. In gewissen Fällen jedoch scheint die Photophobie von einer exzessiven Reizbarkeit der Sehzentren, welche ihrerseits durch den Reizzustand der Meningen bedingt wird, abhängig zu sein. Die definitive Entscheidung dieser Frage muß noch weiteren Untersuchungen überlassen werden.

Komplizierende Läsionen, die wahrscheinlich unabhängig sind von der Paralyse des N. abducens.

Es sind hierhier vor allem die Läsionen des Warzenfortsatzes and die umschriebenen, um die Sinus herum gelegenen Abszesse zu erwähnen.

Abgesehen von den 4 Fällen die letal endeten, wurde unter ว3 Fällen nur 24 mal die Antrektomie in der Warzengegend gemacht und zwar 4 mal lediglich zu diagnostischen Zwecken, wegen der Gefahr, welche die Symptomatologie infolge des Auftretens der Paralyse des N. abducens darbot. Nun konnten also Läsionen des Warzenfortsatzes konstatiert werden und es fehlten die Symptome einer Reaktion desselben in fast zwei Dritteilen der Fälle oder besser in 62 Prozent.

Die Bedeutung der in Rede stehenden Komplikation in der Pathogenese der Paralyse des N. abducens wird noch dadurch herabgesetzt, daß die Alterationen, welche bei der Antrektomie des Warzenfortsatzes, nachgewiesen werden konnten, oft von nur geringer Bedeutung waren, indem nur blasse Granulationen in ge- 
ringer Quantität, wenig Eiter in der Tiefe des Antrum usw. angetroffen wurden. Diese Tatsachen beweisen in evidenter Weise, dah die Komplikation von Seite des Warzenfortsatzes nur eine sekundäre Bedeutung hat.

Die Radikaloperation am Mittelohr wurde im ganzen $5 \mathrm{mal}$ ausgeführt und zwar $3 \mathrm{mal}$ bei chronischen, 2 mal bei akuter Otitis, in den letzteren Fällen sekundär nach der einfachen Antrektomie, wegen Persistenz der Eiterung in der Trommelhöhle.

Ein um den Sinus gelegener Absze 3 ist 6 mal unter 20 Fällen von Warzenfortsatzoperationen, bei denen positive Befunde erhoben werden konnten, wahrgenommen, also in 30 Proz. Es ist dies ein Prozentsatz der jedenfalls als ein hoher angesehen werden muß. Doch mulb bemerkt werden, daß in diesen Fällen der Abszeb auf die dura mater des Sinus und des unmittelbar angrenzenden Gebietes beschränkt war und sich nicht längs des oberen hinteren Randes der Pyramide, gegen deren Spitze fortsetzte. ${ }^{1}$ )

Die Exploration der mittleren Schädelgrube wurde $5 \mathrm{mal}$ ausgeführt, und zwar $4 \mathrm{mal}$ mit negativem Erî́nge, d. h. ohne daß irgend eine Alteration an der oberen Fläche des Felsenbeins, anf dem tegmen antri, dem tegmen tympani und auf der entsprechenden Strecke der dura mater hätte nachgewiesen werden können.

Blob in einem Falle (Goris) entdeckte man Granulationen und es konnte ein kleiner Knochensequester, scheinbar von der Spitze der Pyramide entfernt werden.

Eine andere Komplikation von der, obgleich nur selten, Erwägung gemacht wird, ist die Reizung (Spasmus des musc. orbicularis oculi) oder Paralyse des 7 . Gehirnnerven auf der Seite des erkrankten Ohres (4 Fälle auf 53 , ungefähr 7 Proz.). Das Auftreten dieser Paralyse ist in verschiedener Weise gedeutet worden, aber sie wird höchstwahrscheinlich durch eine Läsion des Nerven in seinem intratympanalen Verlaufe bedingt wie es bei gewöhnlichen Mittelohrentzïndungen vorzukommen pflegt und ist also nicht vom Mechanismus des Entstehens der Paralyse des N. abducens abbängig. Übrigens spricht auch das seltene Vorkommen derselben dafür, daß sie bloß als eine Komplikation angesehen werden müsse.

1) Die Fälle von tiefen extraduralen Abszessen, die sich bis zur Spitze des Felsenbeines ausdehnen und die Paralyse des N. abducens veranlassen, sollen später erörtert werden. 
Beachtenswert ist ein Fall, (Terson I) in welchem, auf derselben Seite, an welcher eine Otitis mit typischen Erscheinungen auftrat, vorher eine akute Otitis vorbanden war, die sich damals mit einer Paralyse des Facialis, die 6 Wochen andauerte, kompli. zierte. Blob 2 mal wird als Komplikation Labyrinthentzündung angeführt, die sich aber, wie es scheint, durch keine bestimmten klinischen Symptome auszeichnete, und hauptsächlich auf Grund des operativen Befundes diagnostiziert wurde, bei welchem einmal eine Fistel des äußeren halbzirkelförmigen Kanales, das andere mal eine Dislokation des Steigbügels und die Eröffnung des ovalen Fensters konstatiert wurde. Eine genaue Prüfung dieser zwei klinischen Beobachtungen erlaubt jedoch nicht mit Sicherheit auszuschlieben, dak es sich blof um akzidentelle bei der Operation erfolgte Läsionen handelte. Jedenfalls ist es sicher, dab in 90 Proz. der typischen Fälle gar keine Alteration des Jabyrinthes vorkam. Dies ist ein Umstand, der für die Frage der supponierten reflektorischen Genesis des Paralyse des äußeren geraden Augenmuskels von großer Bedeutung ist.

Wenn wir nach der Prüfung der wichtigsten klinischen Merkmale der uns beschäftigenden Krankheit, den Mechanismus untersuchen, der dieselbe hervorbringt, dann finden wir, dal dies. bezïglich verschiedene Hypothesen geäuBert worden sind.

Die Verschiedenheit der Meinungen kann zum Teile nur durch den Mangel des pathologisch-anatomischen Materials, an dem es in früherer Zeit fehlte, erklärt werden. Die Zunahme der klinischen Beobachtungen, das Studium mehrerer Fälle mit tödlichem Ausgange, der Vergleich mit pathologisch-anatomischen Läsionen in analogen Fällen gestatten aber gegenwärtig ein ziemlich sicheres Urteil abzugeben.

Wir wollen zunächst die Theorie eines reflektorischen Ur* sprunges der Augenparalyse priffen, eine Theorie, die viele Anhänger hatte und auch heute noch von mehreren Autoren, wie Bonnier, Geronzi, und für gewisse Fälle auch von Lombard, vertreten wird. Die engen anatomischen Beziehungen, welche zwischen dem Gehörlabyrinth und dem Augenmuskelapparat bestehen, der Einflub, den nach unseren physiologischen und klinischen Kenntnissen Reizungen des nicht akustischen Teiles des Labyrinthes auf die Bewegungen des Auges ausiben, haben zu der Meinung gefuhrt, daß auch die Paralyse des $N$. abducens als eine vom Labyrinth ausgehende reflektorische Erscheinung angesehen werden müsse. Bei genauer Prüfung der 
Tatsachen kann jedoch gar kein triftiger Grund für diese Anschauung festgestellt werden.

Die motorischen Erscheinungen an den Augen, welche bei physiologischen oder abnormen Reizungen des Labyrinthes beobachtet werden können, kommen konstant gleichzeitig an beiden Augen vor. Es sind Bewegungen von Nystagmus in den verschiedensten Richtungen, und in Fällen von sehr intensiven Reizungen bemerkt man eine konjungierte Deviation beider Augen. Symptome von Paralyse werden unter den reflektorisehen Erscheinungen restibulären Ursprungs nicht angegeben und am allerwenigsten solche bloß an einem Auge, wie es bei den uns beschäftigenden Fällen vorzukommen pflegt. Auch die von Bonnier zitierten vereinzelten Fällen ändern an dieser Regel nichts.

Man muß in Erwägung ziehen, daß es sich oft um verwickelte Fälle handelt, die nur schwer gedeutet werden können, ferner daB bloß der einzige Fall, den Bonnier als Paralyse des N. abducens auf Grundlage einer Otitis anführt als unseren typischen Fällen analog angesehen werden kann, daß aber der reflektorische Ursprung dieser keineswegs nachgewiesen worden ist. Der Fall von Bonnier, obwohl der Autor nur wenige detaillierte Angaben darüber machte, ist in unserer Statistik doch unter Nummer 8 in der II. Kategorie angeführt.

Ein anderer entscheidender Grund, der in den in Rede stehenden Fällen eine Pathogenese durch Reflex von Seite des Labyrinthes auszuschließen erlaubt, ist der, daß Läsionen und überhaupt Erscheinungen von Seite des Labyrinths bloß in ganz außergewöhnlichen Fällen angegeben werden, hingegen in der größten Mehrzahl derselben ausdrücklich erwähnt wird, daß das Labyrinth rollständig intakt war.

Außerdem muß berücksichtigt werden daß Alterationen in der Beweglichkeit der Augenmuskeln, welebe in Fällen einer Läsion oder auch einer einfachen Reizung des Labyrinthes vorzulommen pflegen, auch bei anderen charakteristischen Gleichgewichtsstörungen, namentlich bei der aufrechten Stellung, beim Gehen, beim Springen usw. auftreten können; es sind dartum Brechneigung, Erbrechen, Schwindelanfälle etc. vorhanden. Wir haben gesehen, daß die Schwindelanfälle, welche in einigen der Fälle angegeben werden eher auf Störungen der Motilität der Augenmuskelu, auf die Diplopie, als auf restibuläre Reizung bezogen werden können. 
Auch ist es auffallig in Anbetracht der Frequenz der eiterigen Erkrankungen des Labyrinths, die infolge von eiterigen Mittelohrentzündungen entstehen, daß bei denselben von dem Vorhandensein einer isolierten Paralyse des N. abdncens keine Erwähnung gemacht wird, während im Gegenteil Symptome von Motilitätsstörungen beider Augen angetroffen werden.

Auch die Analyse der einzelnen Symptome erlaubt die Ausschließung der Hypothese des reflektorisehen Ursprungs der Paralyse. So z. B. die lange Dauer der Paralyse des muse. rectus externus, die, wie wir gesehen haben, Wochen und Monate lang bestehen kann, ferner der gradweise Schwund der Paralyse, der zuweilen auch durch exakte Messungen konstatiert wurde. Auch in jenen wenigen Eällen, in welehen nach Elimination der Ursachen einer Eiterretention in der Trommelhöhle, die Paralyse aufhörte, und beim erneuten Auftreten von Retentionserscheinungen, die Paralyse rezidivierte, konnte man beobachten, daß die funktionellen Veränderungen des $\mathrm{N}$. abducens immer erst nach einer gewissen Zeit einsetzten und die Funktionsfähigkeit desselben nie plötzlich zurückkehrte, wie zu erwarten wäre, wenn es sich nur um eine reflektorische, nur nervöse Erscheinung handeln würde.

Daß die Paralyse des $\mathrm{N}$. abducens nicht auf Grund einer einfachen Reizung des Labyrinthes erklärt werden könne, wird bewiesen durch das Auftreten der Augenstörung in einer gewöhnlich späten Periode der Otitis, d. h. in einer Periode, in welcher die Symptome des akuten Stadiums der Otitis und zuweilen der" begleitenden Entzündung des Warzenfortsatzes in Abnahme begriffen oder fast geschwunden sind. Ein Parallelismus zwischen dem Verlaufe der Otitis und der Augenstörung ist nur selten vorhanden; in den meisten Fällen aber fehlt derselbe und häufig persistiert sogar die Paralyse, wenn die Otitis schon aufgehört hat. Dies dentet darauf hin, daß zwischen den beiden Prozessen nur eine unmittelbare Beziehung statt bat.

Auch die Prüfung der anderen wichtigeren Symptome spricht nur wenig zu Gunsten der Annahme eines reflektorischen Ursprungs. Es bandelt sich um eine akute Otitis, deren hoher Grad durch die Intensität und außergewöbnliche Persistenz der Sohmerzen, zuweilen durch Komplikationen von Seiten des Warzenfortsatzes und durch perisinuöse Abszesse, durch das Fieber usw. angezeigt wird. Nicht selten sind anch andere Symptome vorhanden welche, wie Erbrechen, Entzïndung des Sehnerven, Zunahme des Druckes des liquor cerebro-spinalis usw., eine schwere 
Reaktion von Seite der Gehirnhäute andeuten oder, wie die Neuralgien des $V$. Paares, die Reizung des Oculomotorins auf einen Krankheitsherd an der Spitze der Pyramide hindeuten.

Wie könnten diese Symptome durch die Hypothese eines rein nervösen reflektorischen Ursprungs der Paralyse des N. abducens erklärt werden?

Man dachte an eine Entzündung des N. abducens, toxischen, oder rheumatischen oder syphilitischen Ursprungs. Wir miissen jedoch diese aetiologischen Momente in unseren Fällen auf.Grund von klinischen Erfahrungen ausschlieben, ohne leugnen zu wollen daß übrigens der N. abducens ebenso wie andere Nerven solchen Alteration unterliegen könne. Was besonders die Syphilis betrifft, so wurde in einigen der von mir beobachteten Fällen auch eine spezifisehe Kur eingeleitet; aber obne daß damit ein Resultat erzielt worden wäre. Alles spricht also dafur, daß es sich um Läsionen handelt, die sekundär nach der Ohrerkrankung auftreten und den N. abducens in einer Region treffen, wo er von anderen Nerven isoliert liegt.

Betrachten wir zu diesem Behufe den Nerven von seinem Ursprungskerne an und verfolgen wir ihn in seinem Verlanf auf der Gehirnbasis bis zum Eintritte in die orbita.

Die Möglichkeit einer isolierten Paralyse des N. abducens durch Kompression seines Kernes im bulbus, infolge einer Steigerung des intrakraniellen Druckes bei seröser Meningitis, die durch eine Ohrentzündung bedingt wird, wurde von Brieger (12) zugegeben, der in einem seiner Fälle beobachtete, dab die Paralyse nach der Lumbarpunktion regelmäßig verschwand und beim Wiederauftreten des erhöhten Druckes nenerdings zum Vorschein kam.

Es ist jedoch nicht klar, warum der erhöhte Druck gerade auf den Kern des N. abducens und nicht eher auf den Nervenstamme selbst, in der Strecke, wo er isoliert liegt, eingewirkt habe.

Bei Beobachtung des Verlaufes des Nerven an der Gehirnbasis mul man sagen, dab Krankheitsherde in der hinteren Schädelgrube nur schwer den Abducens allein treffen können, ohne auch auf andere ihm naheliegende Nerven, namentlich auf den Facialis und Acusticus einzuwirken. Der Acusticus nun zeigt sich in unseren Fällen immer frei und die Alterationen des Facialis, die übrigens nur in einer sehr kleinen Zahl der Fälle beobachtet wurden, hängen höchstwahrscheinlich von Läsionen desselben in der intratympanalen Strecke seines Verlaufes ab. Zur Erklärung 
der Entstehung einer isolierten Paralyse des N. abducens müssen wir also die Lokalisation der Ursache derselben in einer Gegend annehmen, wo der Nerv auf einer gewissen Streeke wirklich isoliert verläuft. Diese Strecke entspricht, wie wir sehen werden, der Spitze der Pyramide.

Die Beziehungen, welche der N. abducens in dieser Gegend eingeht, sind genau von Dorello (62) beschrieben worden.

Der sulcus petrosus superior verschwindet 4 oder $5 \mathrm{~mm}$ weit von der Spitze des Felsenbeins und es tritt an seine Stelle ein knöcherner Fortsatz von individuell verschiedener Form und Stärke, dessen Spitze nach oben, innen und vorn, d. h. gegen die processus clinoidei posteriores gerichtet ist. Dieser Fortsatz, von Dorello spina sphenoidalis genannt, stellt sich als Fortsetzung der hinteren Lefzedes sulcus petrosus superior dar, der sinus petrosus superior neigt sich, etwas nach außen von der spina, nach vorn, um in den sinus cavernosus einzumünden. Nach einwärts von der spina ist in einer etwas tieferen Ebene eine Depression vorhanden, entsprechend der Spitze des Felsenbeins. Es folgt dann die sutura petrosphenoidalis und schließlieh der änßere Rand der Lamina quadrangularis des Keilbeins, der eine variable Form und Stärlke aufweist. In vielen Fällen zeigt er die Gestalt eines Dritteils eines Kreises, dessen obere Extremität von der Spitze des processus clinoideus post. gebildet wird; zuweilen ist er in zwei Teile geschieden, durch einen kleinen Knochenfortsatz, der etwas unterhalb des processus elinoideus posterior nach auben vorspringt und von Dorello processus dinoideus posterior access, genannt wird. Es entsteht auf diese Weise zwischen der spina sphenoidalis and dem processus clinoideus posterior eine Ausbuchtung, deren Konkavität nach oben und außen gerichtet ist, die aber beim Vorhandensein der Weichteile in einen osteo-fibrösen Kanal umge. wandelt wird und zwar durch Vermittlung eines sehr resistenten fibrösen Bündels, welches ligamentum petrosphenoidale (Grüber) genannt wird.

Um jedoch die anatomischen Verhältnisse dieses Ligamentes zu verstehen, ist es notwendig, die Art und Weise der Befestigung des tentorium cerebelli in seinem vorderen Teile zu berücksichtigen. Es ist das tentorium bekanntlich halbmondförmig, mit einem äußeren Rande, womit es befestigt wird und einem freien inneren, der sogenannte foramen Pacchionii begrenzt. Die Bindegewebsbündel desselben die dem inneren Rande näher liegen, sind oberflächlicher gelegen als die anderen, verlaufen fast sagittal, gehen 
etwas nach außen vom processus elineideus posterior, bis zu den processus clinoidei ant., setzen sich an diese an und verstärken die änBere und obere Wand des sinus cavernosus. Die äuBeren Bündel des tentorium hingegen, welche von außen nach innen verlaufen, inserieren sich erst an die beiden Lefzen des sulcus transversus des Hinterhauptbeins, dann an diejenigen des sinus petrosus superior; die mehr medial gelegenen Bündel sind fast quer gerichtet, verlaufen unter den sagittalen des inneren Randes des tentorium, kreuzen sich + förmig mit denselben, setzen sich an die processus clinoidei posteriores an und beteiligen sich an der Bildung jener fibrösen Lage, welche das Ganglion Gasseri aufnimmt.

Die am tiefsten gelegenen Bündel können als besondere Formationen präpariert werden und bilden das ligamentum petrosphenoidale. Es geht dieses von der spina sphenoidalis des oberen Randes des Felsenbeins aus, inseriert sich an die äuBere Lefzen und an die hintere Fläche der viereckigen Lamelle des Keilbeins etwas unterhalb der processus clinoidei posteriores, an der Stelle der proc. clinoid. post. access., die also nichts anderes als die verknöcherten Anfangsteile des ligamentum petrosphenoidale darstellen. Zwischen dem inneren Teile des oberen Randes des Felsenbeins, dem unteren des äuBeren Randes der viereckigen Lamelle des Keilbeins und dem ligamentum petrosphenoidale befindet sich ein kleiner ungefäbr dreieckiger Raum, mit der Spitze nach außen und mit der Basis nach innen gerichtet. In diesem Raume liegt der $\mathrm{N}$. abducens und der sinus petrosus inferior an der Einmündungsstelle in den sinus cavernosus. Der Abducens legt sich an die untere Wand dieses Stammes an, meistens nahe dem äußeren Winkel, oft beschränkt er sich auf den Winkel, den die spina sphenoidalis mit dem oberen Rande der Pyramide bildet, und ist hier von oben nach unten etwas abgeplattet.

Der sinus spetrosus inferior verläuft erst sehr schief von hinten nach vorn and von auben nach innen, bis er den $N$. abducens der gleichfalls von hinten nach vorn, aber von innen nach außen verläuft, erreicht, ungefähr einen Zentimeter weit hinter der Spitze der Pyramide, uumittelbar vor dem Eindringen in die Dura mater. Nach dem Zusammentreffen der beiden Gebilde, wird die Richtung des Sinus gerade, nähert sich mehr der sagittalen Ebene, kommt über dem Nerven zu liegen und tritt mit ihm in den erwähnten osteo-fibrösen Raum hinein. Hier sind aber die Beziehungen zwischen beiden verschieden, je nach der Lage die der 
Abducens eimnimmt. Wenn dieser den äußeren Winkel des Raumes einnimmt, dann liegt der Sinus auf seiner inneren Seite; bet Lagerung des Nerven mehr gegen die Mittellinie hin liegt hingegen der Sinus iiber demselben und deckt ihn überall mit Ausnahme der unteren Seite.

Ans der genauen Beschreibung von Dorello werden also die engen Beziehungen verständlich, die zwischen dem N. abducens und dem sinus petrosus inferior stattfinden dort, wo der Nerv die Dura mater passiert und durch den erwähnten osteo-fibrösen Raum verläuft, wo er leicht und isoliert komprimiert werden kann. Gleich nach dem Verlassen jenes Raumes macht der Nerv eine leichte Krümmung, dringt in den sinus cavernosus ein und tritt in nahe Beziehungen zu der Arteria carotis.

Angenommen nun, daß die Läsion des Abducens sekundär nach einer akuten eitrigen Ohrentzündung, in der Gegend der Spitze der Pyramide erfolge, wie ist der Mechanismus derselben?

Dorello selbst stellte hierüber eine Hypothese anf, die sich auf die Beziehungen gründet, die der Abducens mit dem sinus petrosus inferior eingeht, während beide durch einen osteofibrösen unnachgiebigen Raum verlaufen. Dorello meint, dab der Sinus von dem jeweiligen Zustande des Gehörorgans beeinflußt werden müsse, da er, nebst anderen Venen die venae auditivae interna (2-3) aufnimmt, die Blut aus dem Labyrinthe und vom Boden der Trommelhöhle sammeln. Die Wände dieser GefäBe erleiden bei eitriger Ohrentzündung einen gewissen Grad von odematöser Schwellung; die entzündliche Reaktion kann, auch wenn eine Thrombose des sinus ausbleibt, doch die Wände desselben ergreifen, und eine Periphlebitis mit Hyperaemie und Ödem der umgebenden Gewebe hervorrufen. Derartige Alterationen können ohne Einflub bleiben auf den N. abduzens bis sie sich aut den mittleren Teil der sinus petrosus inferior beschränkten; sie müssen aber, wenn sie sich auf seine vordere Extremität, die im osteofibrösen Kanal verläuft, ausdehnen, Kompressionserscheinungen hervorrufen, die sich als Parese oder Paralyse des Nerven kundgeben.

Es ist diese Hypothese jedenfalls sehr verfuhrend; allein, es sprechen dagegen anatomische und klinische Gründe, so dab sie in der großen Mehrzahl der Fälle unhaltbar wird.

Vor allem ist das Labyrinth, wie wir gesehen haben, immer in unsern Fällen frei und es ist deshalb auszuschließen, daß Symptome von Entzündung und Retention durch die venae auditivae internae bedingt werden könnten. Was die Venen der 
Trommelhöhle betrifft, so gehören diese zum Gebiet des plexus pharyngeus, der vena meningea media, jugularis interna und externa und haben folglich keine direkten Beziehungen zum sinus petrosus inferior.

Außerdem muß in Erwägung gezogen werden, daß man bei der Annahme, es könnte eine venöse Kongestion, die durch akute Entzündung der Trommelhöhle bedingt wird, auf den Inhalt oder auf die Wandungen des sinus petrosus inferior von EinfluB sein, eine Stase und Oedem bedingen, nicht gut verstehen würde, warum derartige Störungen der venösen Zirkulation sich nicht auch auf den sinus eavernosus und dann auf die vena ophtalmica fortsetzen. Man müßte in derartigen Fällen eine Kongestion der Venen des Augenhintergrundes wahrnehmen. Dies ist aber in der Regel nicht der Fall und es handelt sich in den wenigen Fällen, in welchen Alteration des Augenhintergrundes konstatiert werden konnte, um eine genuine Entzündung des Sehnerven, die höchstwahrscheinlich einer Meningitis serosa zugeschrieben werden müsse.

Die Hypotbese von Dorello würde schließlich die Todesfälle mit Erscheinungen von Leptomeningitis nicht erklären.

Andere Autoren, wie z. B. Morgardi (32) nehmen an, daß sich die Infektion von der Trommelböhle aus auf den carotischen Kanal, dann auf den sinus cavernosus fortsetzt und eine Läsion desjenigen Abschnittes des $\mathrm{N}$. abducens, der im Sinus verläuft and in enger Beziehung mit der Carotis steht, bedingt. Es ist diese ohne Zweifel ein anatomisch vorbereiteter Weg und klinische Erfahrungen zeigen, daß infektiöse sich in der Trommelböhle abspielende Prozesse zu Thrombosen des sinus cavernosus und zum Entstehen einer eitrigen Leptomengitis Veranlassung geben können. Allein eine derartige Pathogenese scheint in unsern Fällen unzulässig zu sein, denn in keinem derselben kam infektiöse Thrombose des sinus cavernosus vor und wie wir sagten, sind auch die Alterationen am Augenhintergrunde nur selten verzeichnet.

Bezuiglich einiger Fälle, in denen in der unmittelbaren Nähe der äußeren Wand des sinus sigmoideus Abscesse vorkamen, könnte man an eine Diffusion von periphlebitischen Alteratiònen längs der Sinus petrosi (des oberen oder des unteren) bis zum sinus cavernosus und an eine konsekutive Läsion des Abduzens im Innern des sinus cavernosus denken. Wir erinnern jedoch daran, daß derartige Abszesse nur selten vorikommen, und daß sogar in einigen typischen Fällen die Integrität des Sinus and 
der Dura mater der mittleren und linteren Schädelgxube durch die explorative Operation erkannt wurde.

Später werden wir sehen, daß extradurale Abszesse welche sich einer Caries des inneren oberen Randes der Pyramide zugesellen, indem sie die Dura mater bis zur Spitze derselben vom Knochen loslösen, den N. abducens erreichen und eine Paralyse hervorrufen können. Allein es handelt sich hierbei um Vorgänge, die verschieden sind von denjenigen, welche die typisehen Fälle der in Rede stehenden Erkrankung kennzeichnen und der Ausgang in Heilung könnte bloß durch geeignete ope rative Eingriffe erreicht werden

Als einzige Erklärungsweise kann also bloß die Diffusion der Infektion von der Trommelhöhle aus nach der Spitze der Pyramide angenommen werden; die Infektion würde den N. abducens an dem Punkte treffen, wo er den osteo-fibrösen Kanal verläßt oder im Kanale selbst. Die anatomischen Wege, die als präformierte angesehen werden müssen, weil die Symptome in den verschiedenen Fällen eine überraschende Einförmigkeit zeigen, können im wesentlichen zweierlei sein: die Infektion verbreitet sich entweder von dem vorderen Teile der Trommelhöhle und von der Tube aus, längs der um die tuba herum gelegenen Knochenzellen, bis zur Spitze der Pyramide oder durch die canaliculi carotico-tympanici auf den carotischen Kanal und dann auf die an der Spitze des Felsenbeins gelegenen Knochenzellen. Der erstere Modus seheint häufiger rorzukommen.

Schon v. Troeltseh hat auf die Bedeutung hingewiesen, welche die pneumatische Zellen, die zuweilen fast über das ganze Felsenbein sich ausdehnen, für die Ausdehnung von infektiösen Prozessen von der Trommelhöhle aus bis zur Spitze der Pyramide haben können. Urbantschitsch (13) beschreibt und bildet $a b$ in seinem Handbuche eine Öffnung im Knochen in der Nähe des ostium tympanicum tubae, an der oberen Seite oder etwas nach hinten, am promontorium. Es führte dieselbe in die in der Nähe der Trommelhöhle und des Labyrinthes gelegenen pneumatischen Räume, die sich oft bis zur Spitze der Pyramide erstrecken. In einem Präparate von Urbantschitseh hatte die Öffnung einen Durchmesser von $21 / 2 \mathrm{~mm}$; die in dasselbe eingefuhrte Sonde drang bis zur Spitze der Pyramide vor und war durch die sehr dünne Knochenrinde hindurch sichtbar. In einigen Präparaten waren in der Knochenrinde kleine Lakunen vorhanden, durch welche hindurch die pneumatischen Zellen mit der Schädelhöhle kommunizierten. 
Bezold (59) sagt, daß die pneumatischen Zellen sich auf das ganze Schläfenbein ausdehnen können, sodaß sie bloß die squama und die vordere Wand des knöchernen Gehörganges frei lassen. Er beschreibt genau auch die in der Nähe der tuba gelegenen pneumatischen Zellen der unteren Wand der Trommelhöhle und diejenigen der tuba, die homolog der bulla ossea der Säugetiere sind.

Sieben man $n$ teilt mit, daß die Peumatisierung des Schläfenbeins bei verschiedenen Individuen stark variiert, und beschreibt Gruppen von peritubären Luftzellen, namentlich mittleren und unteren, die sich nach vorn bis in die Spitze des Felsenbeins fortsetzen. Außer den canaliculi carotico-tympanici sind, wie Siebenmann sagt, ausgedehnte Dehiscenzen vorbanden, durch welche die Trommelhöhle direkt mit dem karotischen Kanal kommuniziert.

Tonietti, der auf mein Ansuchen Untersuchungen an ungefäbr 800 Schädeln des hiesigen anatomischen Museums vornahm, fand, daß an der Spitze der Pyramide nicht selten Lufträume vorhanden waren; in einigen Fällen, in welchen durch irgend eine Öffnung an der Spitze der Pyramide Quecksilber injiziert wurde, drang dasselbe in den karotischen Kanal ein.

Die angeführten anatomischen Eigentümlichkeiten rechtfertigen den Ausspruch, daß in jenen Fällen, in welchen die Pneumatisierung der Schläfenbeine stark ausgesprochen ist, eine präformierte Bahn vorhanden ist, die vom vorderen Teile der Trommelhöhle bis zur Spitze der Pyramide führt. In anderen Fällen, in denen keine anatomische natürliche Babn vorhanden ist, bewirkt doch die pneumatische Struktur der Teile bei entzündlichen Prozessen des Knochens und konsekutiver Zerstörung der Scheidewände, welche die pneumatischen Räume von einander trennen, daß nachträglich Kommunikationen sich ausbilden.

Die Kenntnis dieser Tatsachen klärt viele klinische Eigentümlichkeiten, welche unsere Fälle charakterisieren, auf: die relative Frequenz der Trommelfellöiffnung im vorderen Anteile; die relativ lange Zeit, welche zwischen der ersten Manifestation der akuten Otitis und dem Auftreten der Abduzenslähmung vergeht, da eine gewisse Zeit zur Zerstörung der Septa notwendig ist, die die Lufträume von einander trennen, während die seltenen Fälle ron frühzeitigem Auftreten der Paralyse vielleicht durch die Annahme von präformierten direkten Kommunikationen erklärt werden könnten. Es werden auch die engen Beziehungen erklärlich, welche in gewissen Fällen zwischen der Steigerung und Abnahme 
der Symptome von Retention in der Trommelhöhle einerseits und der Steigerung und der Abnahmeder paretischen Erscheinungen bestehen; die Bilateralität der Läsion des Abducens in Folge des symmetrischen Banes der Schläfenbeine, usw.

Das Auftreten von Komplikationen in der Gegend des Warzenfortsatzes und in der Umgebung des Sinus sigmoideus, welche in gewissen unseren Fällen von Mittelohrentzündungen auftreten, kann vielleicht auch als ein Hinweis auf den praevalierend pneumatisehen Bau des Schläfenbeins gedeutet werden und die ParaIyse des Facialis könnte vielleicht das Bestehen, von Dehiscenzen am Falloppi'schen Kanal andeuten.

Es würde sich also in den typischen, nicht komplizierten Fällen unserer Krankheit um einen infektiösen Prozeb der Trommelhöhle handeln, der sich durch die peritubären pneumatischen Zellen, oder durch diese und den karotischen Kanal hindurch bis zur Spitze der Pyramide fortsetzt. Es entsteht an dieser Stelle ein Krankheitsherd, welcher den Abducens an einem Punkte trifft, wo er den beschriebenen osteofibrösen dreieckigen Raum verläßt. Die Läsion bleibt in der größeren Zahl der Fälle extradural; dies erklärt die relative Gutartigkeit der Fälle und die Möglichkeit einer vollständigen Heilung nach dem Schwunde der akuten Entzündungserseheinungen der Trommelhöhle. Die sehr intensiven Schmerzen, welche von den Kranken in die Schläfengegend und in die Tiefe der orbita verlegt werden dürften auf die Osteitis der Pyramidenspitze und wabrseheinlich auf die umschriebene, der Knochenerkrankung entsprechende Pachymeningitis bezogen werden.

In den Fällen, welche wir zur zweiten Kategorio rechneten, erstrecken sich die der Osteitis der Pyramidenspitze entsprechenden Reizerscheinungen nicht nur auf den Abducens, sondern auch auf die benachbarten Nerven, namentlich auf den Trigeminus und den Oculomotorius; in anderen Fällen erfolgt eine Reaktion von Seite der Hirnhäute, die sich durch die bekannten Erseheinungen der serösen Meningitis kundgibt. Schließlich kommen auch Fälle vor, (unsere dritte Kategorie) in denen die umschriebenen Läsionen der Hirnhäute, durch Diffusion, in Folge von eiteriger Leptomeningitis, zum Tode führen. Der Mechanismus der Entstehung der Symptome scheint in den typischen Fällen auf die beschriebene Weise zu Stande zu kommen. Es kann jedoch geschehen, dab die Osteitis an der Spitze der Pyramide und die Paralyse des Abduzens in anderer Weise sich ausbildet. In solchen Fällen handelt es sich aber nicht um das typische Krankheitsbild und 
es ist wichtig, namentlich mit Rücksicht auf die Therapie und die Prognose, diese Unterscheidung zu machen.

Die Hypothese, welche wir bezïglich der Entstehungsweise der typischen Fälle ausgesprochen baben, wird auch durch pathologiseh-anatomische Befunde bestärkt; in den seltenen Fällen, in welchen der Kranke infolge von Leptomeningitis zugrunde geht, ist natïrlich eine diffuse Ausbreitung der Läsion vorhanden und es kann deshalb die Reihenfolge der Alteration nur schwer mit Sicherheit festgestellt werden.

Von pathologisch-anatomischen Befunden, die sich auf eine Osteitis der Pyramidenspitze beziehen, besitzen wir drei demonstrative Fälle, einen von Noltenius (50), einen andern von Lo mbard (52) (IV. Fall) und einen dritten von mir selbst. Nur der von Noltenius beschriebene Fall gehört unserm typischen Krankheitsbilde an. Eine Reihe anderer Befunde belenchten die verschiedenen Modalitäten der Entstehung der Paralyse des $\Upsilon$. abducens die zwar nicht zum typischen Krankheitsbilde gehören, aber wegen der Differentialdiagnose doch von Wichtigkeit sind.

Der Befund von Noltenius ist von besonderem Interesse:

Mämnliches Individuum, 23 Jahre alt, mit akuter rechtsseitiger Otitis, die konsecutiv nach Angina entstand, mit Fieber. Am 3. Tage trat spontane Perforation des Trommelfells im vorderen Viertel auf. Am 30. Tage der Erkrankang wiederholten sich, nach einer vorübergehenden Periode, in der eine Besserung des Leidens statthatte, die Fiebererscheinungen und es traten deutliche Erscheinungen einer reaktiven Mastojditis auf. Bei der Operation sah der ganze Warzenfortsatz pneumatisch, die Zellen mit Eiter orfullt. Am 2T. April, d. h. 40 Tage nach dem Beginne der Otitis, war der Kranke, mit Ausnahme einer leichten schleimigen Sekretion in der Tubagegend, gebeilt und verlieb das Krankenhaus. Am 25. Jnli wurde in der Chloroformnarkose und vom Gehörgange aus eine Anslöfelung der granulierenden Tubagegend ausgeführt; das Instrument drang in eine Höhle von Erbsengröße ein, die überall von Knochengewebe umgeben war. Am nacholgenden Tage traten starke Schwindelanfälle auf, am 20. Juli Symptome von Meningitis, Schmerzen in der Schläfen- und Lumbalgegend; am 30. Juli Paralyse des rechten Abducens, mit Kopfschmerzen und geringem Sopor, geringer Sekretion aus der Tubagegend. Das Labyrinth, auf das man eingriff, erwies sich normal. Am 3. August trat der Tod ein. Bei der Autopsie wurde eiterige Meningitis an der Gehimbasis und am Rückenmarke vorgefunden; Gehirn und Sinus zeigten keine Alterationen; die Pyramidenspitze hatte kein normales Aussehen; die Dura mater war so stark adhaerent, daß sie bei den Ablösungsversuchen zerrissen wurde. Der Knochen zeigte sich entsprechend dem Dache der knöchernen Portion der Tube nekrotisch so dab die Sondenspitze in denselben eindringen konnte; man sah, daß die Infektion sich von der Trommelhöhle auf die an die erwähnte Portion der Tube gelegenen Luftzellen ausdehnte, hier eine Osteitis hervorrief und dann die Gehimhäute erreichte. Überraschend war, dah die Pyramidenspitze auch auf der linken Seite, fastan derselben Stelle, verfärbt sich zoigte und ähnliche Läsionen wie rechts aufwies, nur weniger intensiv. Der Kranke hatte sich äber jene Seite nie beklagt.

Archiv f. Ohrenheilkunde. 74. Bd. Festschrift. 
Die Beobachtung von Noltenius ist wichtig für die klinische und pathologisch-anatomische Deutung der Läsionen der peritubären Luftzellen und erklärt wie eine Infektion von der Trommelböhle aus sich auf die Pyramidenspitze fortsetzen kann. Man beachte den Reichtum des Schläfenbeins an pneumatischen Zellen und die Doppelseitigkeit der Entzïndungserscheinungen an der Pyramidenspitze.

\section{Lombard (52), IV. Beobachtung.}

Männliches Individuum von 20 Jahren, mit eiteriger akuter Otitis traumatischen Ursprungs, auf der linken Seite. Perforation am 5 . oder 6. Tage. Die anfangs heftigen Kopfschmerzen nebmen nach der Perforation ab, nahmen aber wieder zu und beschränkten sich anf das Auge, auf die Stirn- und linksseitige Scheitelgegend, aber mit außerordentlicher Heftigkeit. Es waren auch Fiebererscheinungen vorhanden. Kleine Perforation im vorderen, unteren Segmente. Paralyse des linksseitigen Abducens; Augenhintergrund normal. Die Zustände verschlimmerten sich in wenigen Tagen und es traten Brechanfälle auf. Dor Kranke starb im Koma nach epileptiformen Anfällen.

Bei der Autopsie konstatierte man eine allgemeine Leptomeningitis. An der Spitze des Felsenbeins war eine ausgebreitete nach der Längsachse des Knochens gerichtete Hobhlung vorhanden, die sich vom tubercnlum occipitale und vom foramen auditivum internum bis zum prozessus clinoideus post. erstreckte. Die Gegend des Ganglion Gasseri war von Eiter eingenommen; der Abducens lag schwebend in dieser eiterigen Masse. Der sinus cavernosus und der canalis caroticus waren normal. Die Schleimhaut der Trommelhöhle zeigte sich angeschwollen, oedematös, und dasselbe Aussehen bot die Schleimhaut der pneumatisehen Zellen des Warzenfortsatzes, die jedoch keinen Eiter enthielten.

Das Felsenbein der anderen Seite war an der Spitze pneumatisiert. Das in die Luftzellen injizierte Quecksilber drang bis in die Trommelhöhle vor, und dies beweist, daß zwischen der Trommelhöhle und den pneumatischen Zellen der Pyramide leichte Kommunikationen vorhanden sind.

Za dem beschriebenen extraduralen Abszesse führte ein Kanal, der in der Substanz des Felsenbeins ausgegraben war und an der oberen Seite der Trommelhöhle ỉber dem aditu\& endete. Der Kanal war für eine dicke Borste durchgängig.

$\mathrm{Zu}$ beachten ist in diesem Falle der Sitz der Perforation im vorderen, unteren Segmente des Trommelfells, außerdem der entzündliche Herd an der Spitze des Felsenbeins, ferner der pneumatische Bau der Felsenbeinspitze quf beiden Seiten und, wie auf der gesunden Seite nachgewiesen werden konnte, die leichte Kommunikation zwischen der Trommelhöhle und den Luftzellen der Felsenbeinspitze. Allerdings wird auch eines Kanales erwähnt, durch welchen die Höhlung des extraduralen Abszesses mit dem aditus tymp. kommunizierte; wahrscheinlich handelte es sich um den canalis subarcuatus, der nach der Beschreibung aber nicht kariös war, so daß die Transmission der Infektion in die Schädelhöhle höchstwahrscheinlich durch die kariös entartete Spitze des Felsensbeins erfolgte. 
Gradenigo. Es bandelte sich in meinem Falle um ejnen Knabcn von 6 Jahren, der wegen Akutwerdens einer doppelseitigen chronischen eiterigen Mittelohrentzitndung mit reichlichen Granulationen die Klinik aufsuchte. Nach Auslöffelung und Entfernung der Granulationen auf der rechten Seite manifestierten sich nach vorhergehender Paralyse des abducens dexter Symptome von Leptomeningitis und es trat rasch der Tod ein. Bei der Autopsie konstatierte man die Zeichen einer diffusen eiterigen Lepto. meningitis mit eingedicktem Exsudat, namentlich in der Keilbeingegend. Am rechten Schläfenbeine war an der Pyramidenspitze ein kariöser Herd vorhanden; der musc. tensor tympani, das Ganglion Gasseri, der Abducens und Oculomotorius lagen im Eiter. Im Antrum war eingedickter Eiter in geringer Quantität vorhanden; das Labyrinth zeigte sich intakt. Auch auf der linken Seite war die Spitze der Pyramide von pneumatischem Bau.

Auch in diesem Falle, der in manche Hinsicht dem von Noltenius analog ist, ist die Leptomeningitis sekundär nach Läsion der peritabären Lufträume der Felsenbeinspitze aufgetreten.

Die beschriebenen pathologisch-anatomischen Befunde bekräftigen die Anschauung über den Mecbanismus der Entzündung auch bei typischen Fällen der Paralyse des N. abducens, die wir auf anatomische und klinische Tatsachen grindeten, d. h. die Anschauung, daß es sich um einen Herd von Osteitis an der Spitze der Pyramide handelt.

Die Paralyse kann aber noch auf andere Weise entstehen, und zwar zunächst infolge eines extraduralen Abszesses am hinteren Rande der Pyramide und an den benachbarten Knochenoberflächen. Es wird, beim Besteben eines derartigen Abszesses und bei Ausbreitung desselben nach vorn, die Dura mater vom Knochen abgelöst und es wird auf den Abducens an der Stelle, wo er an der Dura mater durchtritt, in dem oben beschriebenen osteo-fibrösen Raume, ein Zug oder eine Kompression ansgeübt.

Die Paralyse des N. abducens kann auberdem noch in einer Weise entstehen, die gewissermaßen als eine Art von Mittelweg. zwischen den angegebenen zwei Entstehungsarten angesehen werden kann, nämlich durch eine mehr oder weniger diffuse Osteomyelitis der pneumatischen Zellen des Schläfenbeins, die sich nach vorn bis auf die Spitze der Pyramide erstreckt und auch ausgedehnte extradurale Eiteransammlungen veranlassen kann. In derartigen Fällen ist es nicht leicht zu entscheiden, ob die eine oder die andere Art von Läsionen die Paralyse des Abduzens herbeigeführt hat und es ist gewöhnlich auch das Labyrinth an dem eitrigen Prozesse beteiligt.

Wir wollen kurz mit einigen Beispielen die verschiedenen Formen des in Rede stehenden Prozesses illustrieren. 
1. Tiefer extraduraler Abszeb, der sich naeb vorn gegen die Spitze der Pyramide ausdehnt.

Wir haben gesehen, dab auch in einigen der typischen Fälle extradurale perisinuöse Abszesse als Komplikationen angegeben werden (30 Proz. der Fälle). Allein es handelt sich hierbei um eiterige Ansammlungen, welche auf die hintere Schädelgrube, in der Umgebung des Sinus begrenzt bleiben und nicht mit der Paralyse des $\Delta$ bducens in Zusammenhang gebracht werden können. In den Fällen hingegen, die wir jetzt berücksichtigen wollen, erstreckt sich die eiterige Ansammlung bis gegen die Spitze des Felsenbeines. Die Entwicklungsweise derselben ist verschieden: sie kann entstehen durch Diffussion eines primitiven um den Sinus Sigmoideus herum gelegenen Herdes, durch Ausbreitung von Knochenentzündungen vom Aditus mast. bis zur Schädelfläche des Felsenbeins usw., gewöhnlich durch den canalis subarcuatus hindurch, oder durch Ausbreitung von Läsionen der pneumatischen Zellen, welche in der oberen Wand des knöchernen Gehörganges nnd in der Schuppe des Schläfenbeins enthalten sind.

Bezüglich der Diffusion eines perisinuösen Abszesses gegen die Spitze der Pyramide möchte ich hier einen Fall von Citelli (75) und von Poli (64) anführen.

Citelli (ij) 2. Fall. Mann von 43 Jahren. Rechtsseitige akute Mittelohrentzündung. Spontane Perforation nach einigen Tagen; intensive Sehmerzen am Ohre und an der rechten Kopfseite; Paralyse des Abducens, venöse Hyperämie des Augenhintergrundes. Die Eiterung war geringgradig, die Perforation hatte dis Tendenz sich zu schließen, so daß wiederholt die Paracentese gemacht werden multe. Es traten zuletzt Symptome vo n seiten des Warzenfortsatzes auf. Bei der Operation konstatierte man kleines Antrtm, perisinuösen extraduralen Abszeß, der die Dura mater loslöste und sich nach vorn bis über das tegmen tympani hinaus fortsetzte. Sämtliche Symptome besserten sich allmählich und es trat vollständige Heilung ein.

Poli (64). 16 Jahre altes Mädchen. Doppelseitige chronische Mittelohrentzindung mit intermittierender Eiterung. Am 19. Juni Schmerzen am Kopfe und am rechten Ohre; Fiebererscheinungen fehlten. In der folgenden Nacht traten Brechanfälle auf. Am 23. allgemeine Depression, Temperatur 38,2 , Schmerzen in der Stirn- und Scheitelgegend rechts. Bei der otoskopischen Untersuchung fand man Perforation im hinteren oberen Segmente, Granulationen, die vom Aditus ausgingen; die Warzengegend war bei Druck schmerzhaft; Augenhintergrund rechts normal, links beginnende Papillitis Schwindelanfalle fehlten, Kontraktur der Nackenmuskeln in geringem Grade.

Am 26. Juni wurde wegen Verschlimmerung der Symptome die Antrectomie vorgenommen. Der Sinus war von graulichem Aussehen und thrombosiert. Entsprechend der hinteren Fläche des Felsenbeins, $3 \mathrm{~cm}$ weit von der Trepanationsöffnung war ein $A b s z e ß$ vorhanden. Um diesen aufzudecken, wurde die Dura mater auf eine gewisse Strecke in der hinteren Schädelgrube mittels der Sonde emporgehoben. Am nächstfolgenden Morgen trat Diplopie wegen Paralyse des rechtsseitigen Abducens auf. Nach einer kurzdauernden Besserung verschlimmerte sich wieder in der Zeit vom 29. Juni zum 5. Juli der Zustand der Kranken; es traten Brechanfälle, Sopor auf; die Temperatur war subnormal, Puls 64-66. Explorative Punkturen, 
die einige Male am Temporosphenoidallappen und am Kleinhirn vorgenommen wurden, blieben resultatlos.

Am 8. Juli wurde beim Verbandwechsel längs der hinteren Fläche des Felsenbeins ein extraduraler AbszeB, der sich nach oben und vorn erstreckte, ungefähr $8 \mathrm{~cm}$ tief entleert. Der Eiter war übelriechend, ungefähr $20 \mathrm{cbcm}$. Noch am Tage der Operation kam die Kranke zu sich und nahm Nahrung auf.

12. Juli. Progressive Besserung; die Paralyse des Abducens persistiert jedoch und auch links traten Zeichen von $A$ bducenslähmung anf. Am 20 . wurden die Aboucensbewegungen des Auges deutlicher, erst links, dann rechts; die Quantität des Eiters, die beim Verbandwechsel austritt, nahm ab. Am 26. ist die Abduktionsbewegung auch auf der rechten Seite fast vollständig. Der Augenhintergrund war links normal. Fast vollständigre Heilung an 15. September.

Von besonderem Interesse ist in diesem Falle, daß die Paralyse des Abducens rechts, gleieh nach der Ablösung der Dura mater von der hinteren Oberfläche des Felsenbeins erfolgte, und dah später, als die allgemeinen Symptome schon in Abnahme begriffen waren, wahrseheinlich durch Diffusion der reaktiven Erscheinungen auf die die Pyramidenspitze begleitende Dura mater, auch links Paralyse auftrat. Bemerkenswert ist aucb, mit Rücksicht auf die typischen Fälle, daß die Paralyse nach der Abnahme der extraduralen Eiteransammlung, auf beiden Seiten raseh verschwand.

2. Der extradurale Abszeb, der sich nach vorn, in der Richtung der Spitze der Pyramide ausdehntund die Paralyse des Abducens bedingt, kann von den pneumatischen Räumen der oberen Wand des Gehörganges ausgehen.

Eine interessante Beobachtung in dieser Hinsieht wurde von Strazza (71) gemacht:

Mann von 51 Jahren. Akute Mittelohrentzündung auf der linken Seite. Nach Verlauf von 45 Tagen wurde eine subperiostale Eiteransammlung an der Sehlaffenbeinschuppe konstatiert. Bei der Operation sah Strazza, dal in der Schuppengegend, gleich über dem knöchernen Gehörgange, eine Fistel vorhanden war, die in eine Gruppe von kariösen Luftzellen der oberen Wand des Gehörganges und der Schuppe selbst einmündete. Durch Entfernung derselben wurde die Dura mater entblöBt. Nach einer 10 Tago lang andauernden Besserung der Erscheinungen traten neuerdings intensive Schmerzen und Fieber auf; auch die Eiterung nahm zu. Wiederholter operativer Fingriff. Paralyse des Abducens links. Von Seite des Labyrinthes gar keine Symptome.

Bei der Sondierung in der Richtung des Gehörganges unterhalb der Dura mater drang man in eine kleine Höhlung im Knochen, von der aus Eiter hervortrat. Die pneumatıschen Räume waren zum Teile nach der zweiten Operation noch in Eiterung begriffen; sie wurden ausgelöfelt und drainiert. Fs erfolgte allmähliche Besserung sämtheher Symptome.

3. Die Infektion kann sich vom Aditus, durch den canalis subareuatus und dureh die um das Labyrinth gelegenen pneumatischen Räumehindurch, ausbreiten. Es entstehen in derartigen Fällen gewöhnlich tiefgehende extra- 
durale Abszesse in der Gegend des hinteren-aberen Randes des Felsenbeins und zuweilen erfolgt sekundär eine Infektion der Labyrinthräume durch Erosion der senkrechten halbkreisförmigen Kanäle. Außerdem können Läsionen des Abducens, durch Ausbreitung der Osteomyelitis des Schläfenbeins oder des extraduralen Abszesses nach vorn sich ausbilden.

Lombard (52). 3. Fall. 30 Jahre alter Mann. Er trat ins Krankenhaus wegen Neuralgie des Trigeminus ein mit der klassischen Lokalisation an drei Punkten. Man diagnostizierte eine rechtsseitige akute Mittelohrentzündung ohne Perforation des Trommelfelles. Paracentese, wässrige Eiterung, Fieber. Da die Schmerzen anhielten, wurde später die Antrectomie am Warzenfortsatze ausgeführt. Die diploëtische Apophyse war kongestioniert, Eiterung fehlte. Es trat Diplopie wegen Paralyse der rechtsseitigen Abducens ein mit äußerst intensiven Kopfschmerzen und Fieber. Später Ödem am Augenlide rechts mit Protrusion des Auges, Somnolenz and Delirien in der Nacht.

Die Lumbalpunktion erwies zahlreiche polinukleäre Zellen. Tod.

A utopsie: Diffuse Leptomeningitis, namentlich an der Basis; Sinus cavernosus rechts thrombosiert und eiterig. An der hinteren Seite del Pyramide, rechts, eine Eiteransammlung in einer Aushöhlung der Knochensubstanz des Felsenbeins, die sich in den Aditus und bis zum Sinus cavernosus ausdehnte; der Eiter ergab das Ganglion Gasseni; in der Aushöhlung lag ein Knochensequester von ungefähr $2 \mathrm{~cm}$ Länge, in dem noch Segmente, welche die Lulträume von einander trenten, in vereitertem $\mathrm{Zu}$ stande zu erkennen waren.

Das Felsenbein der entgegengesetzten Seite war pneumatisch; die pneumatischen Zellen erstreckten sich bis zur Dura mater, von der sie bloß durch eine papierdünne Knochenlamelle getrennt waren. Die Infektion breitete sich in diesem Falle von der Trommelhöhle aus, kroch in den pneumatischen Mantel des Labyrinths und in die peritubären Lufträume fort, wahrscheinlich auf dem Wege des Canalis subarcuatus.

\section{Noch viel komplizierter sind die folgenden zwei Beobach-} tungen von $\mathrm{Muck}$ (11) und von mir selbst.

Muck. 48 Jahre alter Mann. Akute Otitis Ende Dezember, mit Schmerzen, die sich auf eine Gesichtsseite ausdehnten. Keine Eiterung am Ohr. Am 28. Januar Parazenthese; Ausfluß von geringer Quantität Eiter. Es erfolgte eine voribergehende Besserung der Symptome, aber nach 8 Tagen traten neuerdings Schmerzen auf. Wiederholung der Parazenthese. Am. 7. Februar Antrektomie, bei der eine Schleimansammlung in geringer Quantität und wenige Granulationen angetroffen wurden. Am 18. Februar traten wieder Schmerzen ein. Parazenthese. Am 15. April wurde in Chloroformnarkose die Kommunikation zwischen Antrum und Mittelohr erweitert. Am 18. Juni Fieber. Radikaloperation, die im Antrum und im Mittelohr Granulationen konstatieren ließ.

20. Juni. Viel Eiter, der vom hinteren unteren Teile der Trommelhöhle, von einer hinter dem Facialwulst gelegenen Stelle herkam. 24. Juni. Erweiterung der Fistel hinter dem Facialwulst durch Entfernung des Pyramidenwinkels und Entblößung der Dura der mittleren und hinteren Schädelgrubo. Sinus normal. 27. Juni. Paralyse des Abducens links. Nystagmus in horizontaler Ebene beim Blick nach der rechten Seite. Pupille und Augenhintergrund normal. Die Sonde dringt in der Trommelhöhle nach vorn und oben $1 \mathrm{~cm}$ weit in einem fistelartige Gange vor. Es tritt ein Retropharyngealabszeß anf, der inzidiert wurde. Heilung. Bei der funktionellen Hörprüfung wurde Defekt der Perzeption im Labyrinth links konstatiert. 
Die Erkrankung der pneumatischen Räume und des Labyrinths erfolyte in diesem Falle wahrscheinlich vom Canalis subarcuatus aus. Bemerkenswert ist das Vorhandensein des fistulösen Kanals in der Richtung der Felsenbeinspitze. Auch der retropharyngeale Abszeß weist auf das Bestehen eines infektiösen Herdes an der Spitze der Pyramide hin.

Gradenigo. Francesco F, 28 Jahre alt. Rechtsseitige akute Otitis mit geringer Exsudation und Kopfschmerzen, namentlich anf der rechten Seite. Fieber. Schweres Allgemeinbefinden. Rascher Verfall der Kräfte.

Nystagmus in horizontaler Ebene, hanptsächlich beim Blick nach der rechten Seite. Dyplopie wegen Paralyse des rechtsseitigen Abducens. Pupillen normal. Parese des Facialis rechts. Reichlicher übelriechender Eiter im Gehörgange; Perforation des Trommelfells im vorderen oberen Segmente; die Perforationswände sind granulierend. Warzenfortsatzgegend normal. Bewegungen des Halses schmerzhaft. Paracenthese, dann Antrektomie. Das Antrum war tief und mit Eiter erfüllt; die Sinuswand granulierend; kein deutlicher extraduraler Abszeb. Nach der Operation schwand die Paralyse des Abducens, während die des Facialis unverändert blieb. Der allgemeine Zustand besserte sich bedeutend und gradweise, aber der Eiter blieb immer reichlich und übelriechend. Später schwand auch die Paralyse des Facialis und das Fieber. Der Kranke verlieb das Bett. Drei Wochen nach der Operation jedoch verschlimmerte sich wieder der Zustand; es entstand ein Fistelgang, der im hinteren Teile des Felsenbeins nach oben und vorn vertief.

Es wurde die Fistel in der Narkose erweitert.

Labyrinth-Taubheit. Beim Verbandwechsel traten Schwindelanfälle und Gleichgewichtsstörungen auf.

Wegen Anhaltens der Verschlimmerung der Zustände des Kranken wurde die Radikaloperation ausgeführt. Wir fanden, daß die Trommelhöhle von zum Teil organisierten Granulationen erfüllt war. Der Fistelgang befand sich in der Gegend des Aditus, gleich hinter dem Nervus facialis; die Sonde drang in der Gegend des Labyrinths in einer Strecke von ungefähr zwei Zentimetern ror. Wir entfernten einen kleinen Knochensequester der Labyrinthwand. Bemerkenswert ist, dal im weiteren Verlaufe im Gebiet des dritten Astes des Trigeminus Schmerzen auftraten.

Der Zustand des Kranken verschlimmerte sich rasch. Fieber mit Frost; epileptischer Anfall; Coma. Tod.

A utopsie: Leptomeningitis purnl. der Basis, geringe Exsudation, namentlich in der Keilbeingegend. Die Sinus waren normal, die Dura adhärent am Schläfenbein rechts; bei der Sondierung unterhalb der Dura der Pyramidenspitze, auf der rechten Seite, trat Eiter heraus. Der hintere Teil des Felsenbeins war in eine Abszeßhöhle umgewandelt, in der ein Sequester von schwammigem Baue freilag. Auch die Schnccke war nekrotisch.

Es handelte sich demnach anch in diesem Falle um eine Osteomyelitis mit Nekrose des Schläfenbeins und einer extraduralen Eiteransammlung an der Spitze der Pyramide. Zu beachten ist, daf die Paralyse des Abducens, die wahrscheinlich infolge der Ausbreitung des extraduralen Abszesses gegen die Pyramidenspitze entstand, gleich nach dem ersten operativen Eingriff, wobei ein Abfluß des Eiters an der Operationsstelle ermöglicht wurde, aufhörte und, trotz der sulkzessiven Verschlimmerung der Osteomyelitis und des eiterigen Labyrinths nicht wieder zum Vorscheine kam. 
Von großer Bedeutung bezïglich der Prognose und der Therapie, ist die Unterscheidung zwischen denjenigen Formen der Paralyse, welche in Fallen auftreten, die zu dem typischen Krankheitsbilde gehören und den anderen Formen, welche differenten Ursprunges sind. In den typischen Fällen, in denen keine akzessorischen Symptome und keine Komplikationen vorhanden sind, die wir zur ersten Gruppe rechneten - gründet sich die Diagnose auf das Fehlen von allen Symptomen, die nicht zum charakteristischen Krankheitsbilde gehören, ferner auf die Provenienz der Eiterung vornehmlich aus dem vorderen Teile der Trommelhöhle. Dies wird oft auch durch den Sitz der Perforation im vorderen Viertel des Trommelfells, dureh das Fehlen von Alteration in der Warzengegend, durch das fast konstante Fehlen des Fiebers, die Langsamkeit mit der die Paralyse, ohne äußere Ursachen auftritt und wieder verschwindet und schließlich durch den relativ guten Allgemeinstand des Kranken - was als Kriterium von hervorragender Bedeutung anzusehen ist - bewiesen.

Schwieriger ist die Differentialdiagnose in den typischen Fallen der zweiten und der dritten Kategorie im Vergleiche zu denjenigen, in welchen die Paralyse differenten Ursprungs ist. Will man in denselben mit Sicherheit eine Diagnose stellen, kann sich die Notwendigkeit einer explorativen Operation ergeben. Hierbei muß man nicht nur den Zuständen der pneumatischen Räume des Warzenfortsatzes und des Antrum Rechnung tragen, sondern man wird auch die Gegend des Aditus, die Dura mater des sinus und des angrenzenden Teiles der hinteren Sehädelgrube, vor dem sinus sigmoideus berïcksichtigen, durch Abtragung des hinteren Teiles der Pyramide auf einer kleinen Strecke, um das Bestehen eines tiefen extraduralen Abzesses ausschließen zu können.

Natürlich muß bei solchen Kranken eine genaue Untersuchung: des Nervensystems, des Augenhintergrundes und der labyrinthären Funktionen vorgenommen werden.

Die Therapie variiert je nachdem es sich um typische Fälle handelt oder nicht. In den ersteren, die, wenn keine Komplikationen vorhanden sind, gewöhnlich gutartig verlaufen und zur Heilung gelangen, werden wir bestrebt sein miissen, den Abfluß des Eiters aus der Trommelhöhle möglichst zu erleichtern dureh wiederbolte Parazentesen oder durch Erweiterung der schon bestehenden Perforation; notwendig sind sorgsame Beobachtung des Kranken und sofortige operative Eingriffe sobald irgend ein ver- 
dächtiges Symptom sich geltend macht. Die Antrektomie um den Abfluß des Eiters aus der Trommelhöhle durch die retroaurikuläre Öffnung zu ermöglichen, kann auch beim Fehlen von schweren Reaktionserscheinungen seitens der pneumatischen Zellen des Warzenfortsatzes von Nutzen sein.

Die Kranken mïssen in jedem Falle konstant beobachtet werden, denn es wäre verfehlt, blindlings dem gewöbnlich gutartigen Verlauf der Krankheit Vertranen zu sehenken; man sollte immer an die Eventualität einer tödlichen Leptomeningitis denken, wenn auch eine derartige Eventualität nur selten sich zu realisieren pflegt.

Die Prognose, die gewöhnlich eine gute ist, muß deshalb immer eine reservierte sein.

In den Fällen von Paralyse des Abducens im Gefolge von tiefen extraduralen Abszessen oder einer diffusen Osteomyelitis des Schläfenbeins - die nicht streng zum typischen Krankheitsbilde gehören - muß in energischer Weise operativ vorgegangen werden.

\section{Schlub folgerungen.}

Es existiert ein typisches Krankheitsbild, das im wesentlichen durch eiterige aknte Mittelohrentzïndung, intensive Schmerzen, namentlich in der Schläfen-Scheitelbeingegend der erkrankten Seite und durch Paralyse des $\mathrm{N}$. abducens derselben Seite charakterisiert ist. In Ausnahmefällen kann das Krankheitsbild durch Akutwerden einer chronischen eiterigen Mittelohrentzündung hervorgerufen werden. In ungefähr der Hälfte der Fälle können akzessorische Symptome vorhanden sein, die von einer Reizung des Trigeminus und des Oculomotorius oder der Gehirnhäute abhängen. Als Komplikationen treten zuweilen reaktive Mastoiditis und umschriebene auf den sinus sigmoideus beschränkte extradurale Läsionen auf.

Gewöhnlich tritt vollständige Heilung ein und nur selten erfolgt der Tod mit Symptomen einer diffusen eiterigen Leptomeningitis. Der pathologisch anatomische Prozeb besteht in einer Diffusion der eiterigen Infektion der Trommelhöhle auf die Pyramidenspitze auf dem Wege der peritubären pneumatischen Räume und des canalis caroticus. Der Abducens wird an der Pyramidenspitze angegriffen, gleich nach dem Durchtritte aus der dura mater. Es handelt sich also um eine auf die Pyramidenspitze beschrinkte Osteitis und eventuell um eine entsprechende Pachymeningits. Die extradurale Lokalisation des Leidens erklärt den gewöhnlich gutartigen Verlauf der Krankheit. In einzelnen Fällen aber 
tritt umschriebene seröse oder auch diffuse eiterige Leptomeningitis auf.

Nicht alle Fälle von Abducenslähmung, die im Verlaufe einer Otitis auftreten, gehören dem beschriebenen klinischen Bilde an. Gewisse Formen derselben hängen von verschiedenen pathogenetischen Ursachen $a b$; unter diesen sind die am häufigsten rorkommenden die extraduralen, tiefen Abszesse am oberen Rande der Pyramide, die sich gegen die Spitze derselben ausdehnen und die diffusen osteomyelitischen Prozesse des pneumatischen Mantels des Schläfenbeins, welche die Felsenspitze erreichen. Derartige Fälle gehören natürlich nicht zu den typischen und es muß dementsprechend die Prognose und Therapie in denselben sich verschieden gestalten.

\section{Literatur:}

1883. 1) Bürkner, A. f. O., XIX, 249.

1888. 2) Keller, M. f. O., XXI.

1889. 3) Stix, Z. f. O., XIX.

1895. 4) Meyer, A. f. O., XXXVIII, 258.

1896. 5) Spira, A. f. 0., XLT, 128.

1898. 6) Preysing, Z. f. O. XXXIII, $S$ 8. - 7) Habermann, IIL. Vers. d. Deutschen otol. Gesellschaft, Jena, S. 94. -- 8) Jürgensmeyer, ibid, S. 104. - 9) Katz, ibid, S. 104.

1899. 10) Geronzi, Archivio Italiano di Otologia, ete; VIII, S.1.

1900. 11) Muck, Z. f. 0, XXXVII, 191. - 12) Brieger, Mening. serosa in Euzyklopädie f. Ohr., S. 248.

1901. 13) Urbantschitsch, Lehrb.d. Ohr., S. 452. - 14) Mayo.Collier, Journal of Laryngology ete., XVI, 536. - 15) Dundas Grant, ibid., 537. - 16) Woods, R., ibid., 537.

1902. 17) Pischel, Z. f. O., XL, 273. - 18) Hilgermann, ibid., XL 317. - 19) Sturm und Suckstorf; ibid., XLI, 113.

1903. 20) Török, A. f. 0., XVIT, 188, - 21) d'A jutolo, Archivio Ital. di Otol. etc, XIV, 210. - 22) Körner, Die otitischen Erkrankungen des Hirns etc., III. Ausgabe, Wiesbaden 1903, S. 73. - 23) Goris; Annales mal. oreille, XIX, 64. - 24) Bonnier, Presse med, 16. Dezember 1903.

1904. 25) Gradenigo, Realo accademia di Medicina in Torino, 29. Januar. -. 26) ibid, 22. April. - 27) ibid, 1\%. Juni. - 28) Gradenigo, A. f. 0.. LXII, 255, - 29) Idem, Arehivio ital. di Otologia, XV, 402 . - 30) Idem, Congrès intem. d'Otologie, Bordeaux, 1.-4. Angust 1904. -31) Idem, Annales mal. oreille, $X X X, 120$ - 32) M on gardi, Arehivio Ital. di Otologia ete, $X V, 404 .-38)$ Citelli, ibid., 407. - 34) Rieci, C. A., ibid., 453. - 35) Trifiletti, A., ibid., 484. - 36) Lannois et Ferrand, Soc. franc. dotologie, Mai 1904. - 37) Lubet Barbou, ibidem. - 38) Cheval, ibid. und Presse oto-laryngol. belge, III, 248. - 39) Benoit, Paralysies des muscles oculaires. Thèse de Lyon. 40) Leimer, Z. f. O., 242.

1905. 41) Geronzi, Archivio Ital. di Otol. etc., XVI, 163. - 42) Gradenigo, IX Riunione della Società Ital. di Laring. e Otologia, Ref. in Archivio Ital., XVII, 1906, 323. - 43) Luc. Ref. in Gradenigo 42. - A. Forselles, Uber die frühzeitige Diagnose des Empyems des Warzenfortsatzes. Helsingfors, 1905, und Finska Läk. H andl., 
12. August 1905, 8, S. 136 . - 45) Jack, Laryngoscope, 521. - 46) Pick, 77. Naturforschervers. in Meran, September 1905. -47 ) Gradenigo, ibidem. - 48) Tom masi, Bollettino della Società medica di Lucca, N. 3. - 49, Suñe y Molist, Contribucion a l'estudio clinico de las complicaciones oto-cerebrales. Barcelona, Badia Verl., 1905. 50) Noltenius, Revue hebd. de Laryng., 2. Dezomber 1905.

1906. 51) Terson (Toulouse) und Terson (Paris), Annales mal. or., 15. - 52) Lombard, ibid. 321. - 53) Jacques, ibid. 592. - 54) A t t, M. f. $0 ., 88 .-$ as) Baurowiez, ibid. 535. - 56) Rimini, Archives intern. de Laryngol. etc, XXI, 124 und in Gradenigo Nr. 42. 57) B on nier, ibid. XXIr, 82. - 58) Lannois et Perretiése, ibid. 798. - 59) Bezold, Lehrb. d. Ohr., 8. - 60) Siebenmann, Ana. tomie d. Sinnesorgane von Schwalbe. - 61) Hastings, Archives of Otology, 1 - 62) Dorello, Atti della Clinica ot.-laring. di Roma, per l'ann. 1905, Tipografia Campidoglio, Roma. - 63) Barr Stottart, Journal of Laryngol. etc., März 1906, und Glasgow med. Journ., August 1906. - Poli, Rimnione della Società Ital. di Laring. e Otol., Milano, 1906, September. - 65) Gradenigo, ibidem.

1907. 66) Cozzolino, Giorn. intern. della Scienze mediche, S. 20, und in Chíerici, La Pratica Oto-rino-lanngojatrica, Milano, April 1907. 67) Bellotti, L'Ospedale Maggiore di Mílano, Februar 1907. 68) Schwarzk opf, Sammelreferat, Zentralblatt f. Ohr., Bd. V, Heft 5. - 69) Baratoux, Archives intern. de Laryog. ete. - $701 \mathrm{Cecca}$ roni, Archivio Ital. di Otologia etc., XVIII. - 71) Strazza, ibid. - 72) Poppi, ibid. - 73) Modestini, ibid. - 74) Tommasi, ibid. - 75l Citelli, ibid. 